\title{
Evaluating atrial fibrillations through strange attractors dynamics
}

\author{
Andrei Zala ${ }^{1}$, Dan Dimitriü, Maricel Agop ${ }^{3,4}$, Dan N. Tesloianu ${ }^{5}$, Maria L. Cobzeanu 6 , \\ Bogdan M. Cobzeanu', Marcela C. Rusu ${ }^{3}$ and Vlad Ghizdovat ${ }^{8}$ \\ ${ }^{1}$ Electrical Engineering Department, "Gheorghe Asachi" Technical University, Iasi, Romania \\ ${ }^{2}$ Faculty of Physics, "Alexandru Ioan Cuza" University, Iasi, Romania \\ ${ }^{3}$ Physics Department, "Gheorghe Asachi" Technical University, Iasi, Romania \\ ${ }^{4}$ Academy of Romanian Scientists, Bucuresti, Romania \\ ${ }^{5}$ Cardiology Clinic, „Sf. Spiridon" Clinical Emergency Hospital Iasi, "Grigore T. Popa” University of Medicine and Pharmacy, \\ Iasi, Romania \\ 6 „Sf. Spiridon” Clinical Emergency Hospital Iasi, “Grigore T. Popa” University of Medicine and Pharmacy, Romania \\ ${ }^{7}$ Clinical Recovery Hospital Iasi, "Grigore T. Popa" University of Medicine and Pharmacy, Iasi, Romania \\ ${ }^{8}$ Biophysics and Medical Physics Department, Faculty of Medicine, "Grigore T. Popa" University of Medicine and Pharmacy, \\ Iasi, Romania
}

\begin{abstract}
Nonlinear dynamics is nowadays widely employed in the study of biological phenomena. In such context, taking into account that abnormal heart rhythms display chaotic behaviours, in our opinion, the specific attractor dynamics can constitute a method for evaluating various cardiac afflictions. By using mathematical procedures specific to nonlinear dynamics we devise a new method for evaluating atrial fibrillations. Using data from ECG signals, we construct strange attractors corresponding to the phase space, specific to the analyzed signals. We show that their dynamics reflect abnormal heart rhythms. The skewness and kurtosis values are in accordance with pulse rate distributions from histograms of the analyzed signals. The Lyapunov exponent has positive values, close to zero for normal heart rhythm and with values over one order of magnitude higher in the case of fibrillation crises, highlighting a chaotic behavior for cardiac muscle dynamics. All the employed statistical parameters were calculated for a total of 5 cases (ECG signals) and statistical correlations were made using Python programming language. The presented results show that by applying nonlinear dynamics methods for analyzing the heart electrical activity we can obtain valuable information regarding fibrillation crises.
\end{abstract}

Key words: ECG - Atrial fibrillation - Strange attractors - Lyapunov exponent - Histogram

\section{Introduction}

Almost 60 years ago, Noble developed a first cardiac cell model based on modified Hodgkin-Huxley equations describing the long-lasting action and pace-maker potential

Electronic supplementary material. The online version of this article (doi: 10.4149/gpb_2021016) contains Supplementary Material.

Correspondence to: Maricel Agop, Physics Department, "Gheorghe Asachi” Technical University, Prof. Dimitrie Mangeron Rd., No. 59A, 700050, Iasi, Romania

E-mail: m.agop@yahoo.com of the Purkinje fibers of the heart (Noble 1960). Since then, several nonlinear models of electrophysiological dynamics were developed with increasing complexity and specificity (a review of these models can be found in (Fink et al. 2011; de Godoy 2016; Nayak et al. 2018; Vagos et al. 2018)). These models suggested the use of nonlinear method to investigate the electrical behavior of the heart. In their pioneering works, Guevara et al. (1981), Ritzenberg et al. (1984) and Glass and Mackey (1988) introduced nonlinear approaches to heart rhythm analysis and provided evidence of nonlinear behavior in the electrocardiogram (ECG). Methods borrowed from the theory of chaos control were successfully applied for

(c) The Authors 2021. This is an open access article under the terms of the Creative Commons Attribution-NonCommercial 4.0 International License (https://creativecommons.org/licenses/by-nc/4.0/), which permits non-commercial use, distribution, and reproduction in any medium, provided the original work is properly cited. 
the termination of the repolarization alternans to prevent alternans-induced ventricular tachycardia of fibrillation (Christini et al. 2001a, 2001b), termination of ventricular tachycardia (Ripplinger et al. 2006) and control of electrical turbulence in the heart (Luther et al. 2011).

The heart rate dynamics is one of the most investigated physiological phenomena. A lot of mathematical methods were proposed and applied to heart-rate time series for evaluating the heart-rate variability: power spectrum, autocorrelation function, capacity dimension, correlation dimension, Lyapunov exponents, Hurst exponent, reconstructed phase-space analysis, detrended fluctuation analysis, bifurcation analysis, recurrence plot, approximate entropy, sample entropy, Kolmogorov-Sinai entropy etc. An extensive description of these methods and how they were applied can be found in (Acharia et al. 2007; Krogh-Madsen and Christini 2012; Nayak et al. 2018; Henriques et al. 2020). The Task Force of the European Society of Cardiology and the North American Society of Pacing Electrophysiology (1996) suggested standards for the quantification of heart rate variability, including indices for time and frequency domains.

The nonlinear dynamics investigation of the electrocardiogram time series can offer important information also for other physiological processes than those specific to cardiology. Thus, experimental results revealed that the heartbeat rate signal present a low-dimensional chaotic state during an epileptic seizure, while before and after the seizure event it presents a complex, aperiodic behavior (Su et al. 2008). Also, Young and Benton (2015) associated heart rate complexity with ratings of mood, focused attention reaction times, inhibition, and decision time, concluding that nonlinear rather than linear methods of summarizing the heart rate times series offers a novel way of relating brain functioning and behavior.

Current basic methods for evaluating atrial fibrillation (AFIB) are resting ECG for diagnosis, and ECG Holter monitoring over 24 hours for cases in which the resting ECG is not conclusive but there is a high suspicion of atrial fibrillation or cases in which fibrillation can be seen on the resting ECG but it cannot be determined if this is permanent or appears only in paroxysm (Camm et al. 2009; Iaizzo 2015). Also, an ECG Holter can establish the minimum and maximum ventricular frequency in the course of 24 hours, and if other heart rhythm disorders can be found for the patient in question (atrial flutter, ventricular extrasystoles etc.). If the ECG Holter recordings are again inconclusive, but the patient suffers from palpitations and a neurotic affliction is out of the question, another device can be this time subcutaneously implanted - an ILR (Implantable Loop Recorder) which allows for heart rhythm monitoring for as long as three years and from a distance, even by using smartphone applications. A high risk of AFIB can be supposed if premature atrial complexes can be identified on the resting or Holter ECG recordings (Sawhney et al. 2009). A final method involves the supervised triggering of AFIB by subjecting the patient to an exercise stress test (treadmill test), especially for patients with physical effort palpitations (Walraven 2010).

Although the nonlinear dynamics and complex system theory provided important information on many aspects cardiology, the translation of therapy predictions based on nonlinear systems analysis into clinical practice is still reduced (Krogh-Madsen and Christini 2012). The existing methods must be extended, and new methods must be proposed to improve the performance of clinical applications.

In this paper we devise a new method for evaluating atrial fibrillations, by using mathematical procedures specific to nonlinear dynamics (strange attractors, skewness, kurtosis, histograms, Lyapunov exponent etc.). When compared to the above presented methods for evaluating AFIB, it is our opinion that this method could potentially estimate AFIB risk with a higher accuracy, being less time consuming then a Holter ECG, with a lower health risk than an exercise stress test, and incomparable cheaper than an ILR.

\section{Methods}

The analyzed electrocardiograms were downloaded from the PhysioNet database (PhysioNet). This allows all researchers to access a free collection of physiological signals (PhysioBank), recorded from a wide range of patients, as well as specialized software for viewing and analyzing them. It is supported by the National Institute of General Medical Science (NIGMS) and the National Institute of Biomedical Imaging and Bioengineering (NIBIB), and free access is made in accordance with ODC Public Domain Dedication and License v1.0. Existing resources are made available to stimulate current research in the domain of studying complex biomedical and physiological signals.

The analyzed signal has the following traits:

- 10-hours recording time

- sampling time of $4 \mathrm{~ms}$, sampling rate of 250 recordings/s

- a total of 9205760 points

- amplitude between $-0.6 \mathrm{mV}$ and $0.9 \mathrm{mV}$

These signals have been analyzed using statistical and mathematical nonlinear procedures. In this regard, we operated with median, variance, skewness, kurtosis, and the largest Lyapunov exponent (Arce 2004; Nayfeh and Balachandran 2004). The employed procedures are presented in Supplementary Material A. Moreover, we made correlations between these previous mentioned parameters for a total of 5 analyzed cases (ECG recordings). The detailed methods are presented in Supplementary Material B.

The statistical analyses were performed using Origin Pro, version 9.6.5.169. Shapiro-Wilk test (Shapiro and Wilk 1965) was used to check the normality of the distribution of 
measured data. When normality was rejected (as expected in the cases where the skewness is different from zero), two alternative data distributions were tested, namely log-normal and gamma, using the Anderson-Darling test (Anderson and Darling 1952). If the Anderson-Darling test rejects both distributions, according with the extensive analysis performed by Kula et al. (2020), the median is more appropriate to be considered in data with unknown distribution to characterize the central tendency of data (real mean values). The variability of the data is described by the interquartile range (IQR) $\mathrm{Q}_{1}-\mathrm{Q}_{3}$, which determine the $50 \%$ confidence interval around the median.

\section{Results}

In Figure 1 the graphical representations of ECG fragments ( $5 \mathrm{~s}$ duration) corresponding to pre-crisis, first AFIB
(AFIB 1), atrial flutter (AFL), second AFIB (AFIB 2) and post-crisis, respectively, are shown for the analyzed ECG's presented in Methods.

Let us mention that AFIB represents an abnormal heart rhythm, characterized by rapid and irregular beats. Paroxysmal AFIB is a form of tachycardia, manifesting in the case in which the ventricular electric activity is disorganized and very rapid. The ventricles have very rapid contractions, which makes them inefficient (Iaizzo 2015). In this way, a "pulse deficit" can occur, a discrepancy between the central and peripheral pulses, the latter being lower. The atrial flutter (AFL) is an abnormal heart rhythm, which begins in the ventricular chambers (Sawhney 2009). When it first appears, it is often associated with a high cardiac frequency (over 100 beats/minute), being classified as a type of supraventricular tachycardia.

For the case shown in Figure 1, we observe that in precrisis and post-crisis the pulse is normal. In the first AFIB,

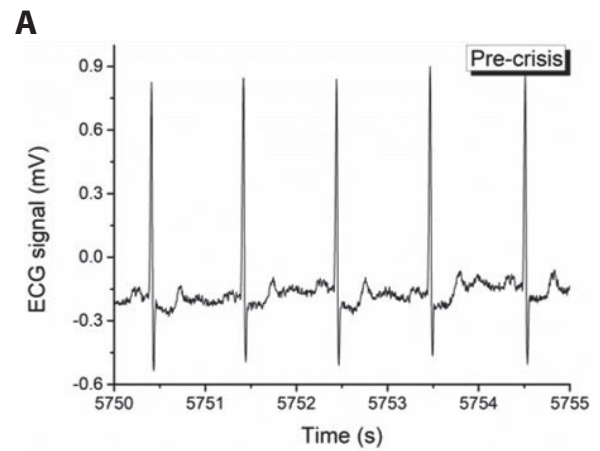

B
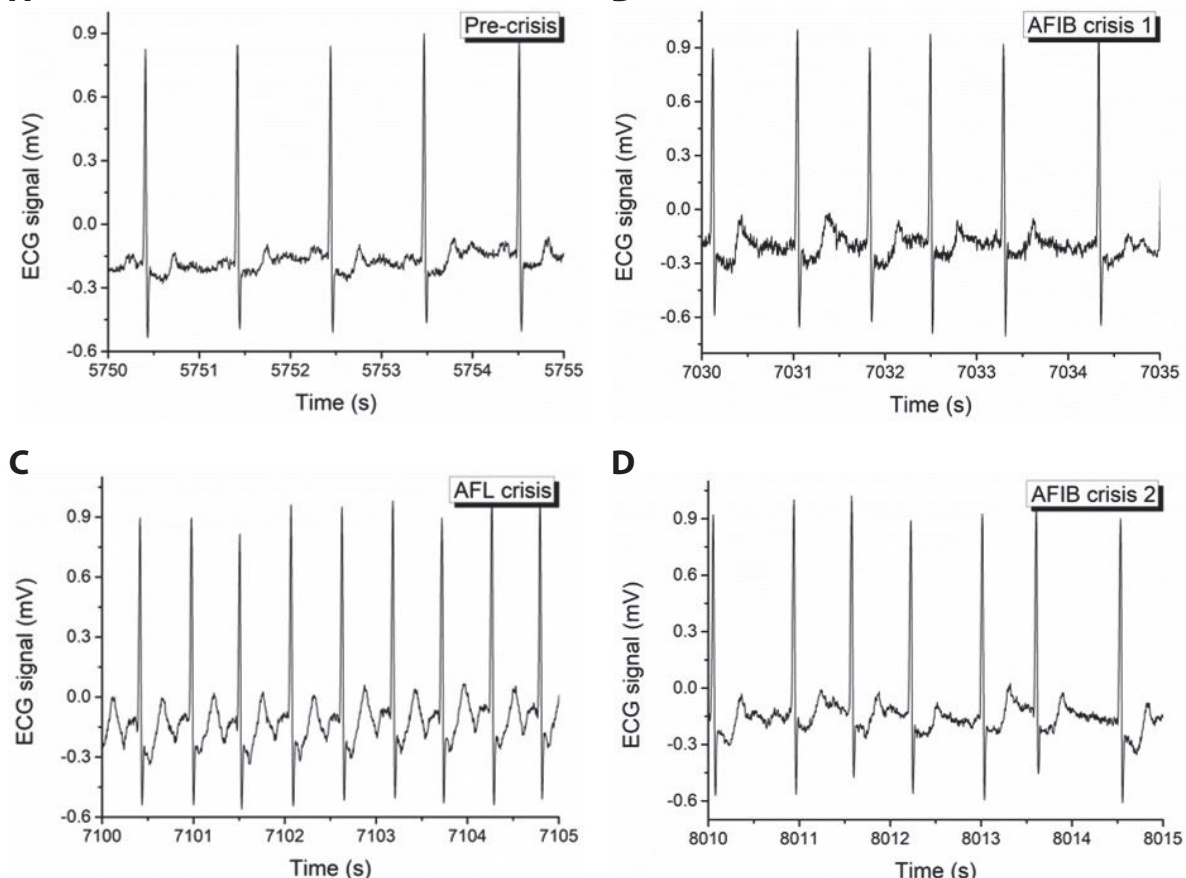

D
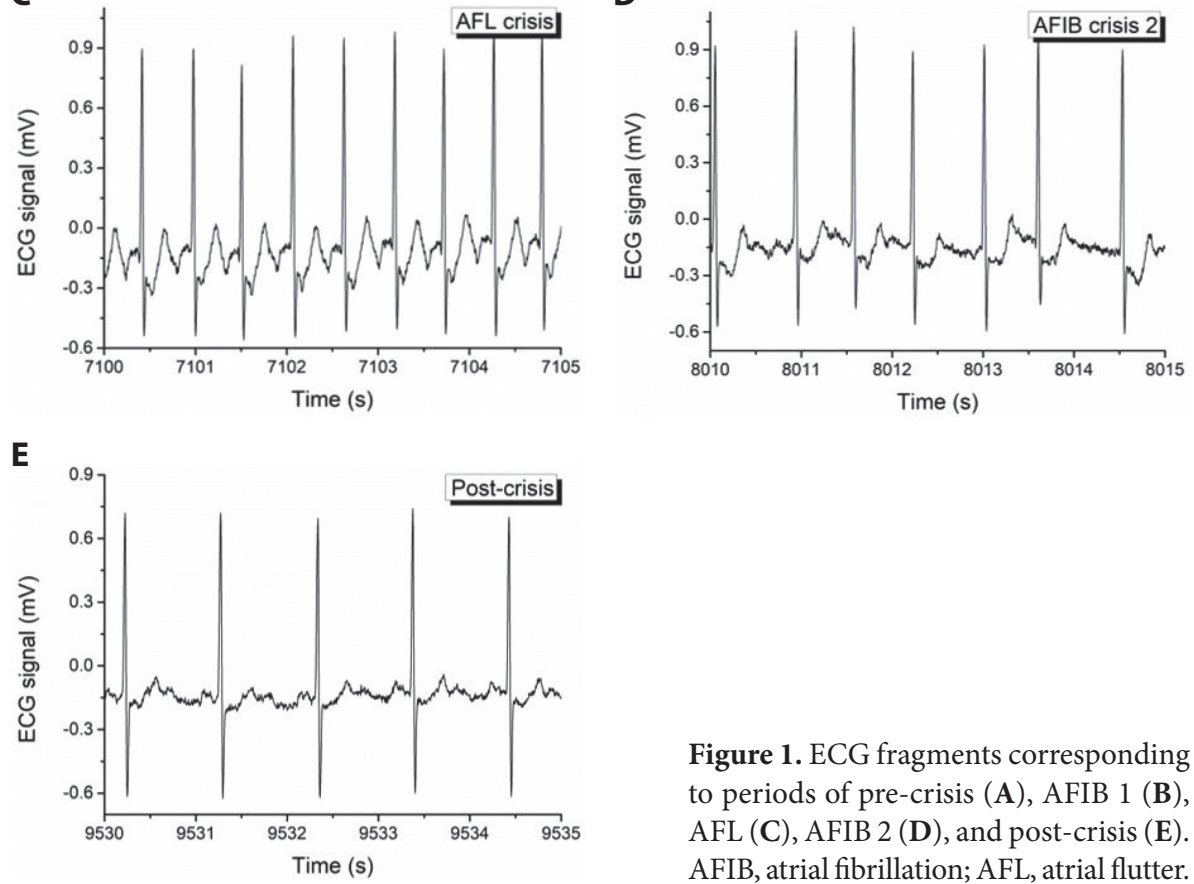

Figure 1. ECG fragments corresponding to periods of pre-crisis (A), AFIB 1 (B), $\operatorname{AFL}(\mathbf{C})$, AFIB 2 (D), and post-crisis (E). AFIB, atrial fibrillation; AFL, atrial flutter. 

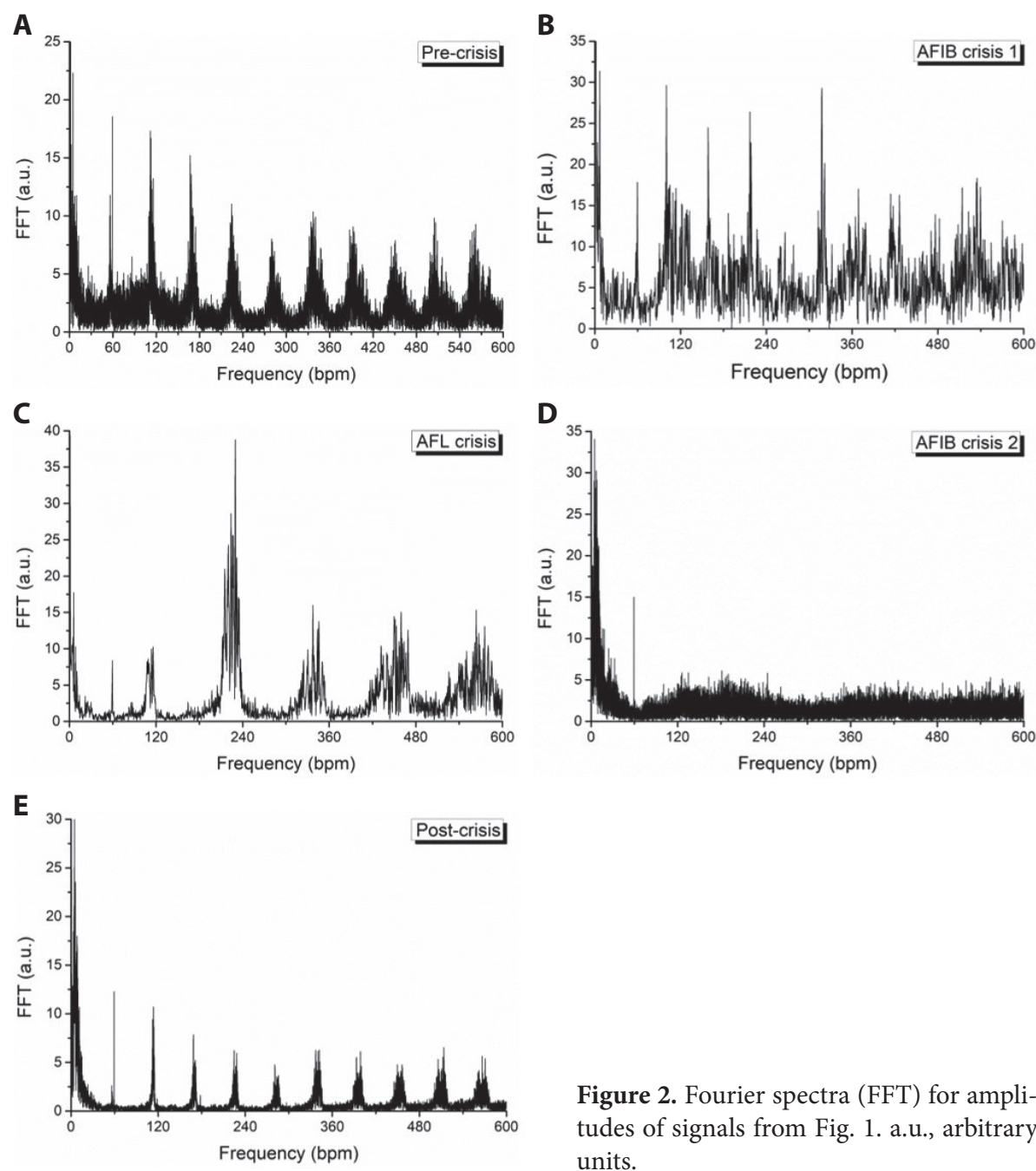

heart rate increases slowly, then it shows a rapid increase in AFL, slowly decreasing afterwards in the second AFIB.

In the following, the results obtained by applying the mentioned nonlinear mathematical procedures are presented.

The Fourier spectra shown above reveals to us the following: i) during pre-crisis (especially when compared to post-crisis), the signal shows a detectable noise, meaning that heart beats start to slowly become irregular, anticipating a crisis; ii) the graphs for AFIB crisis 1 and AFIB crisis 2 display the chaotic behaviour of the heart (heart rate) during these crisis episodes. In atrial flutter, the heart's upper chambers (atria) beat too quickly. This causes the heart to beat in a fast, but usually regular, rhythm. This is confirmed by the specific Fourier spectrum. In post-crisis, the noise starts to decrease, thus showing a return to a relative normal and regular heartbeat (Fig. 2).

Figure 3 shows the systems dynamics attractors in the phase space reconstructed trough the delay time method, determined using the auto-correlation function.

Figure 2. Fourier spectra (FFT) for amplitudes of signals from Fig. 1. a.u., arbitrary units.

We must highlight the clear difference between the geometry of attractors from Figure 3, corresponding to the two atrial crises (AFIB 1 and 2), and also the differences between pre-crisis and post-crisis. This suggests that a defibrillator was used to restore normal heart rhythm.

The analysis of the $1 / \mathrm{R}-\mathrm{R}$ interval, corresponding to a cardiac cycle, highlights these three crises, two atrial fibrillations and one flutter fibrillations - see Figure 4. For further details see Supplementary Material A.

It can be seen that the first AFIB lasts for about $2 \mathrm{~min}$, the atrial flutter crisis is about $8 \mathrm{~min}$, and the second AFIB is much longer, lasting for about $30 \mathrm{~min}$. In AFIB 1 the pulse varies from $60 \mathrm{bpm}$ to $110-120 \mathrm{bpm}$. AFL shows a higher (about $120 \mathrm{bpm}$ and over) but more constant pulse. AFIB 2 is the most severe crisis episode, with rapid pulse variations between normal values and higher values, 120-150 bpm. After this episode, the pulse stabilizes in post-crisis, most probably due to defibrillation, as we have mentioned before. 
The Histogram analysis of signals from Figure 1 is presented in Figure 5.

The Shapiro-Wilk test excluded the normal distribution for all five cases described in Figure 5. Consequently, lognormal and gamma distributions were tested (also shown in Fig. 5). Anderson-Darling test rejected both distributions for all cases. According with the extensive analysis performed by Kula et al. (2020), the median is more appropriate to be considered in data with unknown distribution to characterize the central tendency of data (real mean values).

The statistical analysis of signals is summarized in Table 1.

Figure 6 shows the boxplots corresponding to the five cases, which allow to visually comparing the scatter of the $1 / \mathrm{R}-\mathrm{R}$ interval, numerically described in the second column of Table 1 (Herzig et al. 2017; Caldwell and Cheuvront 2018).

The skewness and kurtosis values are in accordance with pulse rate distributions from histograms in Figure 5 - for further details see Supplementary Material A. The Lyapunov exponent has positive values, close to zero for normal heart rhythm, and with values over one order of magnitude higher in the case of fibrillation crises, highlighting a chaotic behavior for cardiac.

Histogram analysis of signals from Figure 1, presented in Figure 5, shows that, in the beginning, during the precrisis period, the pulse is stable around $60 \mathrm{bpm}$. It tends
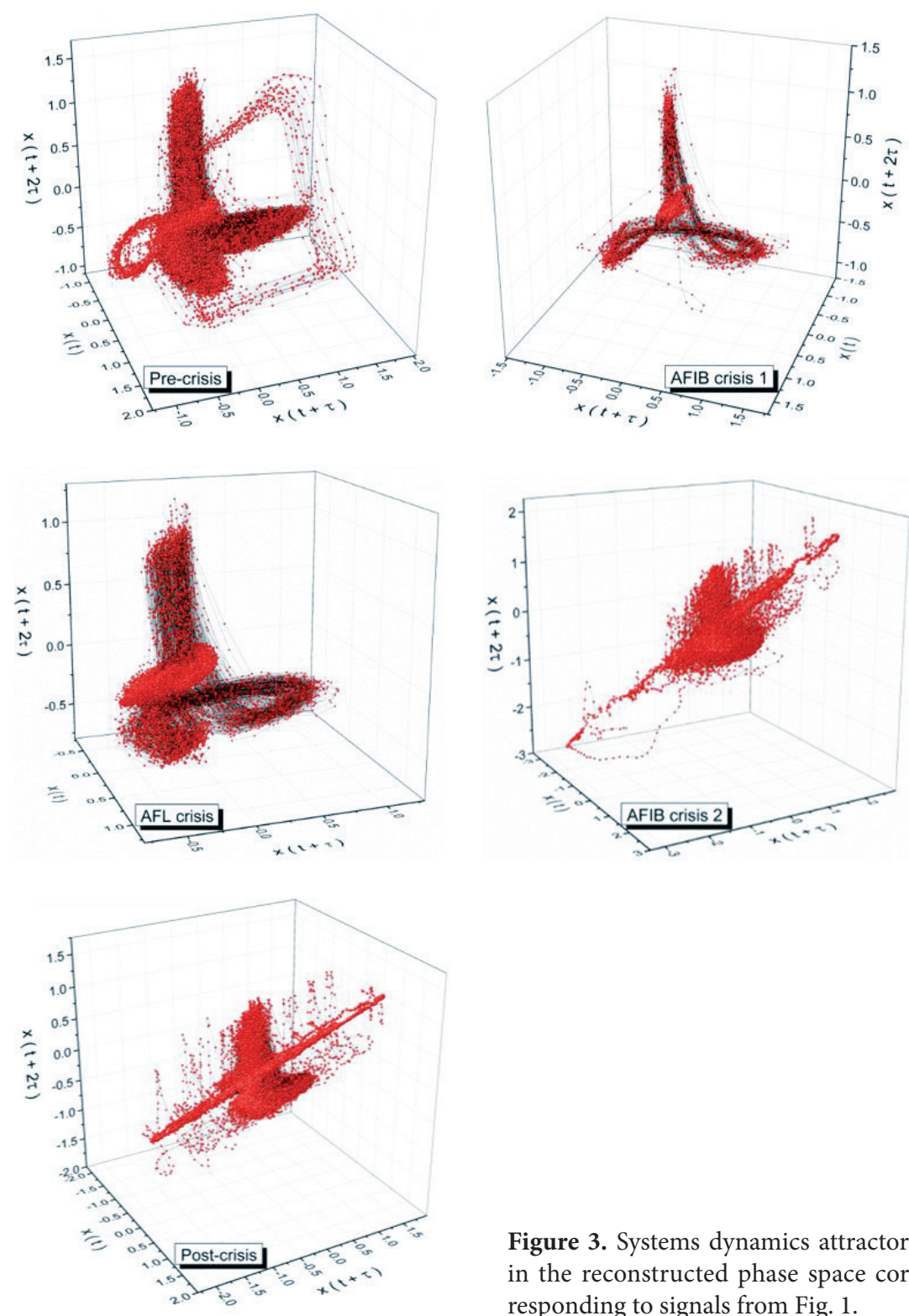

Figure 3. Systems dynamics attractors in the reconstructed phase space corresponding to signals from Fig. 1. 


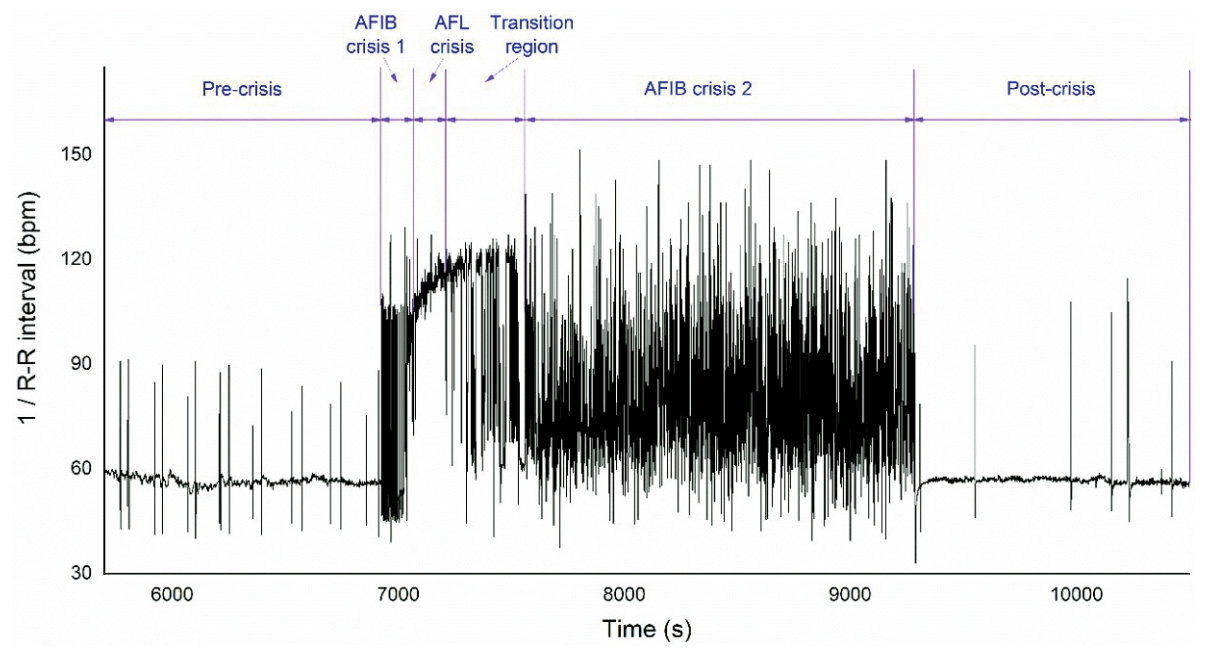

A

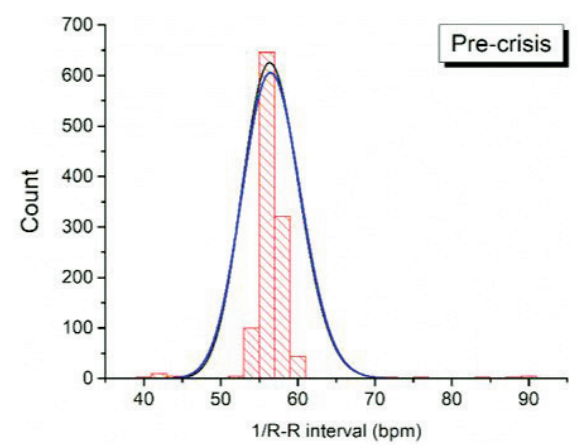

C

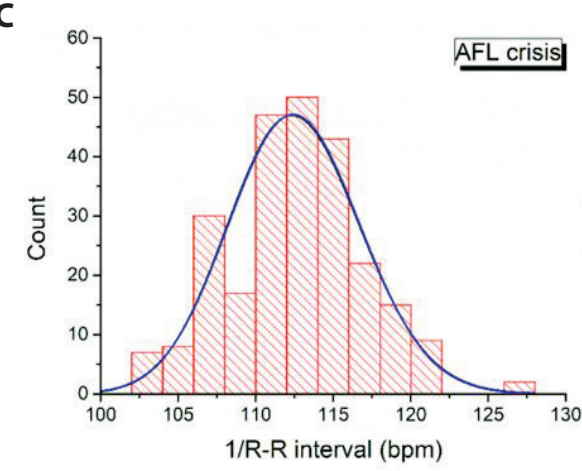

E

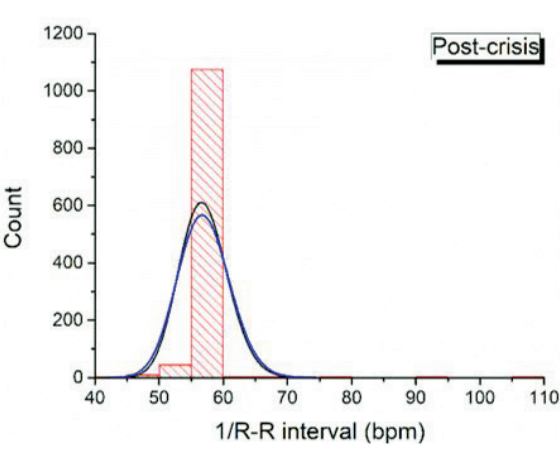

B

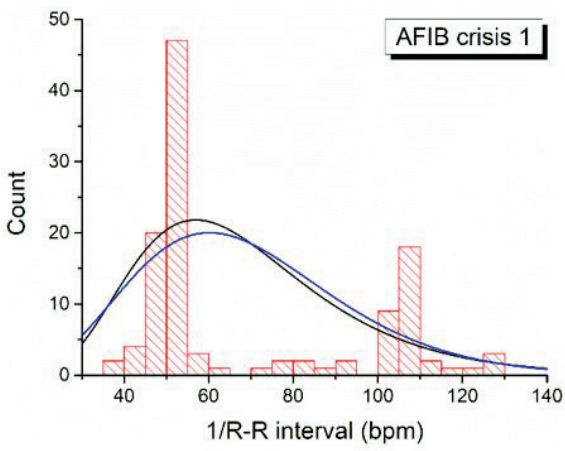

D



F

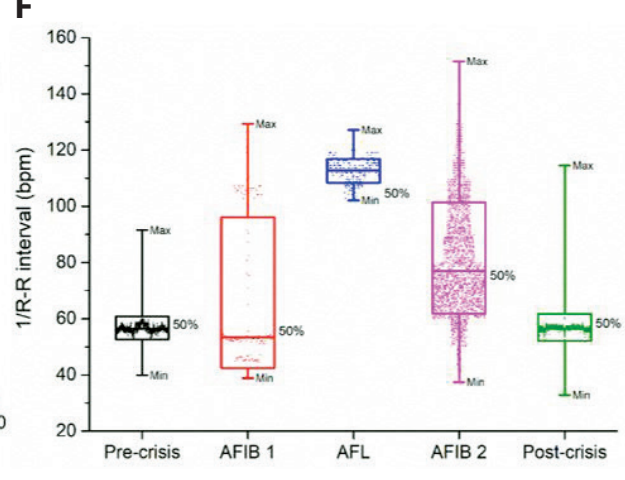

Figure 4. Pulse time variation (1/R-R interval) during fibrillation crises. For abbreviations, see Fig. 1.
Figure 5. Histograms corresponding to periods of pre-crisis (A), AFIB 1 (B), AFL (C), AFIB 2 (D), and postcrisis (E) (1/R-R intervals) and fitting of the data with log-normal (black line) and gamma (blue line) distributions. Boxplots showing the scatter of $1 / R-R$ interval for the five cases (pre-crisis, AFIB 1, AFL, AFIB 2 and post-crisis, respectively) (F). The box outline corresponds to IQR, while the horizontal solid line marks the position of the median. For abbreviations, see Fig. 1. (See online version for color figure.) 
Table 1. Statistical values associated with heart rate variations (1/R-R interval)

\begin{tabular}{lcrrrc}
\hline Signal & $\begin{array}{c}\text { 1/R-R interval median }(\mathrm{bpm}) \text { with the } \\
\text { interquartile range } \mathrm{Q}_{1}-\mathrm{Q}_{3}\left(\mathrm{P}_{25}-\mathrm{P}_{75}\right)\end{array}$ & $\begin{array}{c}\text { Variance from } \\
\text { median }\end{array}$ & Skewness & Kurtosis & $\begin{array}{c}\text { Largest Lyapunov } \\
\text { exponent }\end{array}$ \\
\hline Pre-crisis & $56.3909(55.5556-57.4713)$ & 16.9199 & 4.4938 & 37.6779 & 0.013981 \\
AFIB 1 & $53.3807(51.5464-104.1667)$ & 965.6498 & 0.7814 & -1.098 & 0.211145 \\
AFL & $115.3846(112.7941-117.8846)$ & 25.7844 & -0.0359 & 0.462 & 0.082811 \\
AFIB 2 & $76.9231(66.9643-94.9367)$ & 413.4116 & 0.7047 & 0.0456 & 0.138646 \\
Post-crisis & $56.6037(56.1798-57.2519)$ & 22.9663 & 8.2509 & 82.8455 & 0.014529 \\
\hline
\end{tabular}

AFIB, atrial fibrillation; AFL, atrial flutter.

to increase during the first atrial fibrillation crisis, with a maximum of around $110 \mathrm{bpm}$. During flutter fibrillation, the pulse has values between $100-130 \mathrm{bpm}$. The histogram corresponding to the second atrial fibrillation has an approximately Gaussian distribution, with a maximum at 60-80 bpm. During post-crisis the pulse stabilizes, reaching $60 \mathrm{bpm}$.

We must also highlight the fact that, in the case of atrial flutter, there is a pattern of alternating 2:1, 3:1, 4:1 and 5:1 conduction ratio, as can be observed in Figure 7.

Since aberrant conduction may be sustained in AFL, due to the linking phenomenon, the pattern may mimic ventricular tachycardia. In atrial flutter the atrial electrical activity is far less chaotic than in atrial fibrillation, so that assessment of A-V conduction is less difficult. Nevertheless, it is impossible to determine exactly which out of the atrial impulses has been conducted, due to the extremely fast atrial rate: the conducted impulse, indeed, is not always the one that immediately precedes the QRS complex. In the absence of drugs, atrial flutter is usually associated with 2:1 (or, less frequently, 4:1) conduction ratio, being the odd ratios (3:1, 5:1) far rarer (Oreto et al. 1991). Therefore, we can suppose that at the start of AFL specific drugs have been administered, such as anti-arrhythmic medications. These drugs are used to chemically convert atrial flutter to normal sinus rhythm, reduce the frequency and duration of atrial flutter episodes, and prevent future episodes. They are often given to prevent return of atrial flutter after cardioversion.

\section{Discussions}

As it was shown above, in this paper we employed mathematical procedures specific to nonlinear dynamics (in the form of strange attractors, skewness, kurtosis, histograms, and Lyapunov exponent), aiming to devise a new method for evaluating atrial fibrillations. By using data from ECG signals, we constructed strange attractors corresponding to the phase space, specific to the analysed signals. Furthermore, it is shown that their dynamics reflect abnormal heart rhythms.

\section{Summarization of the main results of our study}

a) skewness and kurtosis values are in accordance with pulse rate distributions from the histograms of the analyzed ECG signals;

b) the Lyapunov exponent has positive values, close to zero for normal heart rhythm, and with values over one order of magnitude higher in the case of fibrillation crises, highlighting a chaotic behavior for cardiac muscle dynamics;

c) in the case of atrial flutter, there is a pattern of alternating 2:1, 3:1, 4:1 and 5:1 conduction ratio;

d) the abnormal heart rhythms can be analyzed through strange attractors dynamics in the reconstructed phase space. It results that to each stage of a crisis a specific strange attractor can be associated;

e) it is difficult to establish threshold values for the significant parameters to detect atrial flutter or fibrillation since the transitions from different regimes (as for example normal heart rhythm to atrial fibrillation, or atrial fibrillation to atrial flutter) are not continuous but sudden. After the transition, the significant parameters suddenly increase/ decrease with one order of magnitude or more (see, for example the variance, skewness, kurtosis or Lyapunov exponent).

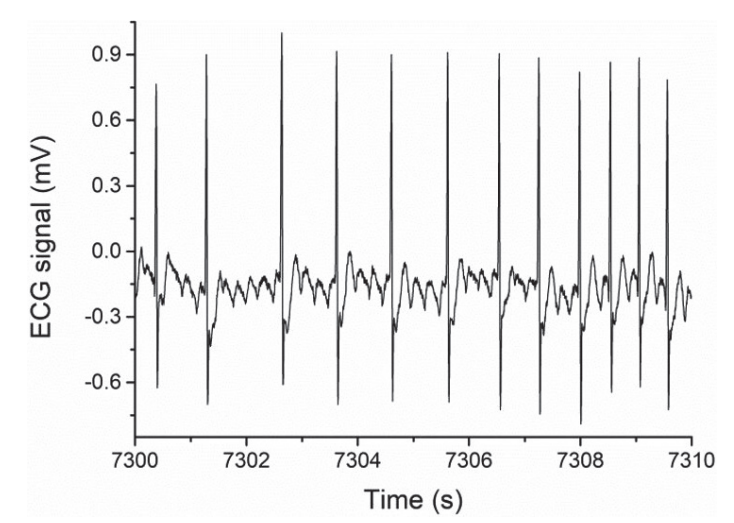

Figure 6. Mixture of 2:1, 3:1, 4:1 and 5:1 conduction ratios during the atrial flutter. 


\section{Validation of our method}

In Supplementary Material B we have selected another 4 ECG signals from the PhysioBank database and calculated the same statistical parameters as in the case presented above. For statistically correlating the results we have used Google Colab ${ }^{\circledR}$.

In Table 2 we present the statistical values associated with heart rate variations (1/R-R interval) for the 5 analyzed cases. The first (uppermost) values are the ones which we thoroughly analyze in the paper.

The statistical analysis of all 5 signals (by using a Python code) reveals the following:

a) strong and very strong correlations can be seen for the pre-crisis and post-crisis stages;

b) fewer strong correlations can be established in the AFIB 1, AFL, and AFIB 2 stages. This could possibly be caused by the fact that in these stages, the signals have a chaotic component also. In such context, further developments of our analysis should also take into account chaotic behaviors (different types of noise).

\section{The novelty of our method}

To the best of our knowledge, no other works have employed all the statistical parameters used in the present paper, for analysing cardiac normal and pathological states. Moreover, we could not find any conclusive data, form a nonlinear dynamics statistical analysis point of view; on the specific pathology we approached: fibrillation with atrial flutter.

\section{Limitations of our method}

We must also mention the fact that the main limitation of our proposed model is that this analysis should be extended to a relative high number of ECG signals, in order to prove consistency. This is mainly due to the fact that, although we are discussing the same pathology, patients' physiologies vary greatly. Although, as it can be seen from Supplementary Material B, the initial results are promising, and they can be considered as a starting point for further developments.

Table 2. Statistical values associated with heart rate variations ( $1 / \mathrm{R}-\mathrm{R}$ interval) for the 5 analysed cases

\begin{tabular}{lccccc}
\hline Signal & $\begin{array}{c}\text { 1/R-R interval } \\
\text { median }(\mathrm{bpm})\end{array}$ & $\begin{array}{c}\text { Variance from } \\
\text { median }\end{array}$ & Skewness & Kurtosis & $\begin{array}{c}\text { Largest Lyapunov } \\
\text { exponent }\end{array}$ \\
\hline Pre-crisis & 56.3909 & 16.919 & 4.4938 & 37.6779 & 0.013981 \\
& 57.0675 & 17.104 & 4.5522 & 38.0171 & 0.014176 \\
& 55.4508 & 17.238 & 4.5066 & 37.1504 & 0.013827 \\
& 65.4882 & 17.492 & 4.6241 & 38.2430 & 0.014519 \\
& 63.6287 & 17.318 & 4.5612 & 34.4752 & 0.014415 \\
\hline AFIB 1 & 53.3807 & 965.6498 & 0.7814 & -1.098 & 0.211145 \\
& 52.7852 & 894.5035 & 0.7829 & -1.114 & 0.215325 \\
& 58.9641 & 984.3088 & 0.7766 & -1.142 & 0.219243 \\
& 75.5347 & 963.1045 & 0.7829 & -1.092 & 0.216212 \\
\hline AFL & 62.3488 & 972.4561 & 0.7663 & -1.090 & 0.199779 \\
\hline AFIB 2 & 115.3846 & 25.7844 & -0.0359 & 0.462 & 0.082811 \\
& 122.4571 & 25.4338 & -0.0357 & 0.4586 & 0.076186 \\
& 131.5822 & 26.0951 & -0.0371 & 0.4719 & 0.081154 \\
& 118.9274 & 24.9041 & -0.0364 & 0.4578 & 0.084715 \\
& 129.6638 & 25.2975 & -0.0362 & 0.4631 & 0.083804 \\
\hline Post-crisis & 76.9231 & 413.4116 & 0.7047 & 0.0456 & 0.138646 \\
& 75.8267 & 422.1173 & 0.7131 & 0.0421 & 0.127831 \\
& 83.9285 & 419.7553 & 0.7216 & 0.0486 & 0.117860 \\
& 90.5721 & 420.4899 & 0.7194 & 0.0457 & 0.138368 \\
& 81.2834 & 436.9258 & 0.7309 & 0.0487 & 0.115384 \\
\hline & 56.6037 & 22.9663 & 8.2509 & 82.8455 & 0.014529 \\
& 59.8244 & 22.9155 & 8.3416 & 82.9449 & 0.014791 \\
& 55.93671 & 23.8193 & 8.5396 & 80.4595 & 0.013367 \\
& 60.1687 & 24.1952 & 8.6676 & 78.9468 & 0.012832 \\
& 23.2470 & 8.4667 & 81.3855 & 0.014120 \\
\hline
\end{tabular}

The first (uppermost) values are the ones which we thoroughly analyse in the paper. 


\section{Conclusions}

The presented results show that by applying nonlinear dynamics methods for analyzing the heart electrical activity we can obtain valuable information regarding fibrillation crises. Our method could be used for developing new models for medical diagnosis and evolution tracking of heart diseases. To this end, we would like to continue our research by analyzing ventricular fibrillations patterns. Taking into account the multi-fractality property of biological structures, a theoretical model based on harmonic mapping on multi-fractal manifolds could prove useful in correlating our results - for further details see (Agop et al. 2008; Colotin et al. 2009; Bujoreanu et al. 2017; Tesloianu et al. 2017).

Authors' contributions. The authors contributed equally to the writing of this paper.

Conflict of interest. The authors declare that there is no conflict of interest regarding the publication of this paper.

\section{References}

Acharia UR, Paul JK, Kannathal N, Lim CM, Suri SJ (2007): Heart rate variability. In: Advances in Cardiac Signal Processing, (Eds. UR Acharya, JS Suri, JAE Spaan, SM Krishnan), pp. 121-165, Springer, Berlin https://doi.org/10.1007/978-3-540-36675-1_5

Agop M, Paun V, Harabagiu A (2008): El Naschies epsilon((infinity)) theory and effects of nanoparticle clustering on the heat transport in nanofluids. Chaos Soliton. Fract. 37, 1269-1278 https://doi.org/10.1016/j.chaos.2008.01.006

Anderson TW, Darling DA (1952): Asymptotic theory of certain "goodness of fit" criteria based on stochastic processes. Ann. Math. Stat. 23, 193-212 https://doi.org/10.1214/aoms/1177729437

Arce GR (2004): Nonlinear Signal Processing: A Statistical Approach. Wiley, Hoboken https://doi.org/10.1002/0471691852

Bujoreanu C, Nedeff F, Benchea M, Agop M (2017): Experimental and theoretical considerations on sound absorption performance of waste materials including the effect of backing plates. Appl. Acoust. 119, 88-93 https://doi.org/10.1016/j.apacoust.2016.12.010

Caldwell AR, Cheuvront SN (2019): Basic statistical considerations for physiology: The journal Temperature toolbox. Temperature 6, $181-210$ https://doi.org/10.1080/23328940.2019.1624131

Camm AJ, Lüscher TF, Serruys PW (2009): The ESC Textbook of Cardiovascular Medicine. 2nd edition. Oxford University Press, Oxford https://doi.org/10.1093/med/9780199566990.001.0001

Christini DJ, Stein KM, Markowitz SM, Mittal S, Slotwiner DJ, Scheiner MA, Iwai S, Lerman BB (2001a): Nonlinear-dynamical arrhytmia control in humans. Proc. Natl. Acad. Sci. USA 98, 5827-5832 https://doi.org/10.1073/pnas.091553398

Christini DJ, Collins JJ, Hall K, Glass L (2001b): Controlling cardiac arrhythmias: The relevance of nonlinear dynamics. In: Handbook of Biological Physics (vol. 4), (Eds. F Moss, S Gielen), pp. 205-227, Elsevier, Amsterdam https://doi.org/10.1016/S1383-8121(01)80009-9

Colotin M, Pompilian GO, Nica P, Gurlui S, Paun VP, Agop M (2009): Fractal transport phenomena through the scale relativity model. Acta Phys. Pol. A 116, 157-164 https://doi.org/10.12693/APhysPolA.116.157

de Godoy MF (2016): Nonlinear analysis of heart rate variability: A comprehensive review. J. Cardiol. Ther. 3, 528-533 https://doi.org/10.17554/j.issn.2309-6861.2016.03.101-4

Fink M, Niederer SA, Cherry EM, Fenton FH, Koivumäki JT, Seemann G, Thul R, Zhang H, Sachse FB, Beard D, et al. (2011): Cardiac cell modelling: Observation from the heart of the cardiac physiome project. Prog. Biophys. Mol. Biol. 104, $2-21$ https://doi.org/10.1016/j.pbiomolbio.2010.03.002

Glass L, Mackey MC (1988): From Clock to Chaos. The Rhythms of Life. Princeton University Press, Princeton https://doi.org/10.1515/9780691221793

Guevara MR, Glass L, Shrier A (1981): Phase locking, perioddoubling bifurcations, and irregular dynamics in periodically stimulated cardiac cells. Science 214, 1350-1353 https://doi.org/10.1126/science.7313693

Henriques T, Ribeiro M, Teixeira A, Castro L, Antunes L, CostaSantos C (2020): Nonlinear methods most applied to heart-rate time series: A review. Entropy 22, 309 https://doi.org/10.3390/e22030309

Herzig D, Eser P, Radtke T, Wenger A, Rusterholz T, Wilhelm M,Achermann P, Arhab A, Jenni OG, Kakebeeke TH, et al. (2017): Relation of heart rate and its variability during sleep with age, physical activity, and body composition in young children. Front. Physiol. 8, 109 https://doi.org/10.3389/fphys.2017.00109

Iaizzo PA (2015): Handbook of Cardiac Anatomy, Physiology, and Devices. 3rd edition. Springer, Cham https://doi.org/10.1007/978-3-319-19464-6_1

Krogh-Madsen T, Christini DJ (2012): Nonlinear dynamics in cardiology. Annu. Rev. Biomed. Eng. 14, 179-203 https://doi.org/10.1146/annurev-bioeng-071811-150106

Kula R, Bébarová M, Matejovič P, Šimurda J, Pásek M (2020) Distribution of data in cellular electrophysiology: It is always normal?. Prog. Biophys. Mol. Biol. 157, 11-17 https://doi.org/10.1016/j.pbiomolbio.2020.05.008

Luther S, Fenton FH, Kornreich BG, Squires A, Bittihn P, Hornung D, Zabel M, Flanders J, Gladuli A, Campoy L, et al. (2011): Low-energy control of electrical turbulence in the heart. Nature 475, 235-239

https://doi.org/10.1038/nature10216

Nayak SK, Bit A, Dey A, Mohapatra B, Pal K (2018): A review of the nonlinear dynamical system analysis of electrocardiogram signal. J. Healthc. Eng. 2018, 6920420, 1-19 https://doi.org/10.1155/2018/6920420

Nayfeh AH, Balachandran B (2004): Applied Nonlinear Dynamics. Analytical, Computational, and Experimental Methods. Wiley-VCH, Weinheim 
Noble D (1960): A modification of the Hodgkin-Huxley equations applicable to Purkinje fibre action and pace-maker potentials. J. Physiol. 160, 317-352 https://doi.org/10.1113/jphysiol.1962.sp006849

Oreto G, Donato A, Satullo G, Luzza F, Calabrò MP, Saporito F, Scimone IM (1991): A-V conduction in atrial fibrillation and flutter. Cardiologia 36, 25-35

Ripplinger CM, Krinsky VI, Nikolski VP, Efimov IR (2006): Mechanisms of unpinning and termination of ventricular tachycardia. Am. J. Physiol. Heart Circ. Physiol. 291, H184-H192 https://doi.org/10.1152/ajpheart.01300.2005

Ritzenberg AL, Adam DR, Cohen RJ (1984): Period multiplyingevidence for nonlinear behaviour of the canine heart. Nature 307, 159-161 https://doi.org/10.1038/307159a0

Sawhney NS, Anousheh R, Chen WC, Feld GK (2009): Diagnosis and management of typical atrial flutter. Cardiol. Clin. 27, $55-67$ https://doi.org/10.1016/j.ccl.2008.09.010

Shapiro SS, Wilk MB (1965): An analysis of variance test for normality (complete samples). Biometrika 52, 591-611 https://doi.org/10.1093/biomet/52.3-4.591

Su ZY, Wu T, Yang PH, Wang YT (2008): Dynamic analysis of heartbeat rate signals of epileptics using multidimensional phase space reconstruction approach. Physica A 387, 2293-2305 https://doi.org/10.1016/j.physa.2007.12.008

Task Force of the European Society of Cardiology and the North American Society of Pacing Electrophysiology (1996): Heart rate variability: Standards of measurement, physiological interpretation, and clinical use. Circulation 93, 1043-1065 https://doi.org/10.1161/01.CIR.93.5.1043

Tesloianu ND, Nedelciuc I, Ghizdovat V, Agop M, LupascuUrsulescu C (2017): A fractal physics explanation for acute thrombotic occlusion in an apparently healthy coronary artery. Anatol. J. Cardiol. 18, 155-157 https://doi.org/10.14744/AnatolJCardiol.2017.7825

Vagos MRSS, van Herck IGM, Sundnes J, Arevalo HJ, Edwards AG, Koivumäki JT (2018): Computational modeling of electrophysiology and pharmacotherapy of atrial fibrillation: Recent advances and future challenges. Front. Physiol. 9, 1221 https://doi.org/10.3389/fphys.2018.01221

Young H, Benton D (2015): We should be using nonlinear indices when relating heart-rate dynamics to cognition and mood. Sci. Rep. 5, 16619 https://doi.org/10.1038/srep16619

Walraven G (2010): Basic Arrhythmias. 7th edition. Pearson, New York

Received: November 11, 2020

Final version accepted: May 7, 2021 


\title{
Supplementary Material
}

\section{Evaluating atrial fibrillations through strange attractors dynamics}

\author{
Andrei Zala ${ }^{1}$, Dan Dimitriu ${ }^{2}$, Maricel Agop ${ }^{3,4}$, Dan N. Tesloianu ${ }^{5}$, Maria L. Cobzeanu ${ }^{6}$, \\ Bogdan M. Cobzeanu', Marcela C. Rusu ${ }^{3}$ and Vlad Ghizdovat ${ }^{8}$ \\ ${ }^{1}$ Electrical Engineering Department, "Gheorghe Asachi” Technical University, Iasi, Romania \\ ${ }^{2}$ Faculty of Physics, "Alexandru Ioan Cuza" University, Iasi, Romania \\ ${ }^{3}$ Physics Department, "Gheorghe Asachi” Technical University, Iasi, Romania \\ ${ }^{4}$ Academy of Romanian Scientists, Bucuresti, Romania \\ ${ }^{5}$ Cardiology Clinic, „Sf. Spiridon” Clinical Emergency Hospital Iasi, “Grigore T. Popa” University of Medicine and Pharmacy, \\ Iasi, Romania \\ 6 „Sf. Spiridon” Clinical Emergency Hospital Iasi, “Grigore T. Popa” University of Medicine and Pharmacy, Romania \\ ${ }^{7}$ Clinical Recovery Hospital Iasi, "Grigore T. Popa" University of Medicine and Pharmacy, Iasi, Romania \\ ${ }^{8}$ Biophysics and Medical Physics Department, Faculty of Medicine, "Grigore T. Popa" University of Medicine and Pharmacy, \\ Iasi, Romania
}

\section{Section A}

\section{Direct analysis of signals. Fourier spectrum}

A signal represents a time recording of a state variable. Signals can be periodic or non-periodic, stationary or non-stationary. A periodic signal is the one that, after a time period $T$, identically repeats itself, being described by a relation like:

$$
x(t)=x(t+k T)
$$

where $k$ is an integer number. In the opposite case, the signal is non-periodic, of a quasi-periodic, chaotic or transitory type. The easiest to identify are transitory signals, because they generally show a short time evolution, due to their rapid dampening.

One of the most important aspects of physiological signals is their oscillatory traits. In this regard, the Fourier analysis is a method of extracting from signal information about its oscillating structure. In the case of a discrete system, the discrete Fourier transform (DFT) is used. A special type of DFT is the fast Fourier transform (FFT), which actually is the most efficient algorithm if we take into account the calculus speed. The FFT representation as a function of frequency is called a Fourier spectrum. In Figure SA1, we show some typical representations of experimental Fourier spectra, corresponding to harmonic, non-linear, quasi-periodic and chaotic signals.

\section{Statistical analysis of signals}

Statistics can provide important information about the nature of the recorded signal. The statistical methods are applied depending on the signal type, after its direct analysis.
The average value of a data set $D$, with $N$ recordings, is defined as:

$M_{D}=\frac{\sum_{i=1}^{N} D(i)}{N}[D(1)+D(2)+\ldots+D(N)] / N$

The median is the value separating the higher half from the lower half of a data set.

The standard deviation is a statistical quantity used for evaluating the spread of individual values around the average of values. A low standard deviation shows that the recorded values are close to their average. For a data set $D$, with $N$ recordings and $M_{D}$ the average, the standard deviation $S$ can be calculated as:

$\mathrm{a} S=\sqrt{\frac{\sum_{i=1}^{N}\left[D(i)-M_{D}\right]^{2}}{N}}$

The standard deviation from the median can be calculated with a formula analogous with Eq. A3:

$S D M=\sqrt{\frac{\sum_{i=1}^{N}[D(i)-\text { Median }]^{2}}{N}}$

Variance is used in descriptive statistics, statistical interference, hypotheses testing, Monte Carlo sampling etc. It is defined as the square of the standard deviation:

$\operatorname{Var}=S^{2}=\frac{\sum_{i=1}^{N}\left[D(i)-M_{D}\right]^{2}}{N}$ 

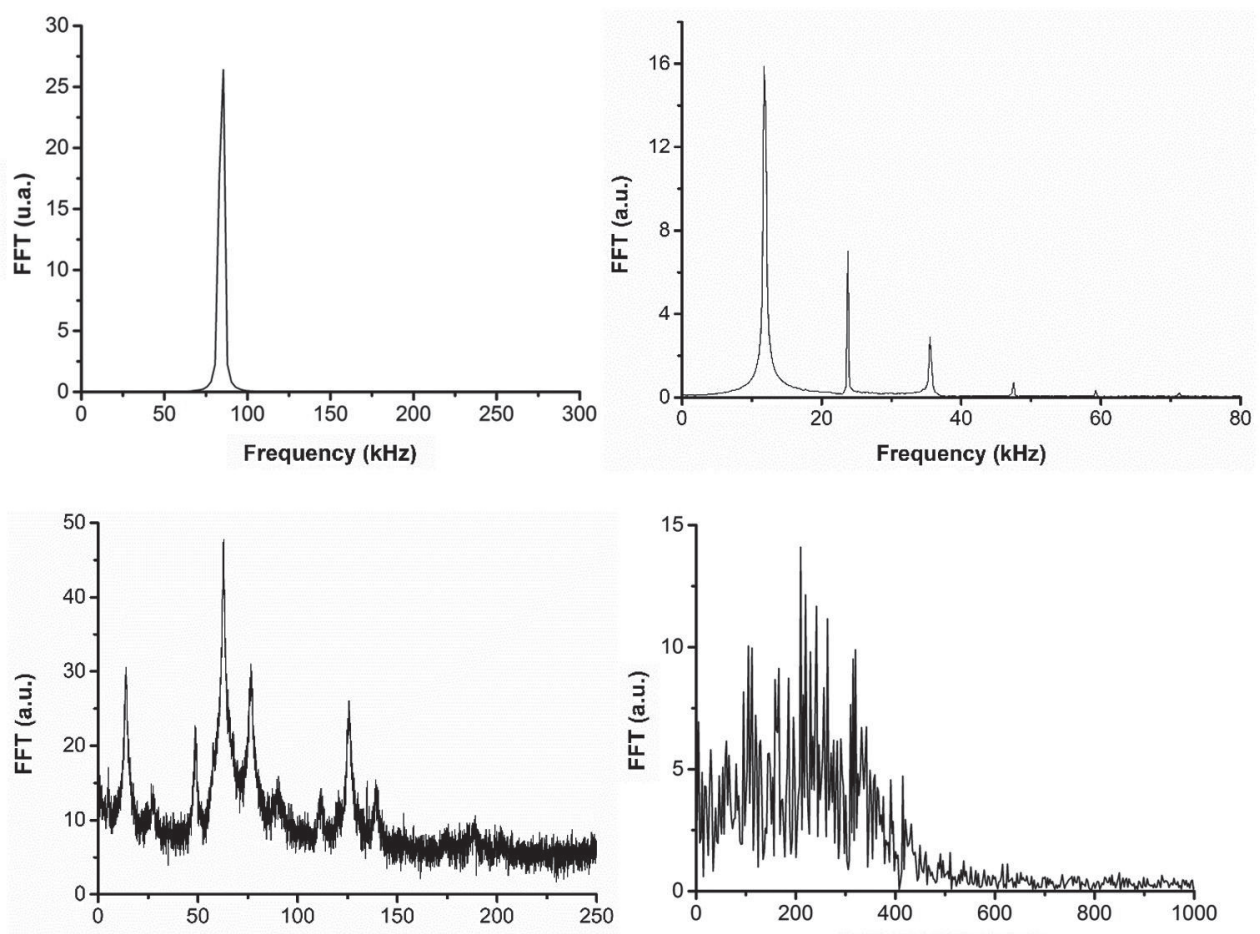

Figure SA1.

Variance from the median is defined as the square of the standard deviation from the median:

$\operatorname{VarM}=S D M^{2}=\frac{\sum_{i=1}^{N}[D(i)-\text { Median }]^{2}}{N}$

In signal analysis, it can be harder to work with signals corresponding to chaotic or nonlinear dynamics, because the characteristic parameters are difficult to establish. In these cases, results interpretation is greatly affected by noise. The type of noise cannot always be determined, and that is why significant progress has been made when a statistical method for separating noise types has been found. Thus, we can use a normal Gaussian distribution and then compare it with the signal of interest. There are two quantities which can be employed for this: skewness and kurtosis.

Skewness is a measure of asymmetry with respect to the normal probability distribution and can be written as:

$\gamma_{1}=\frac{\frac{1}{N} \sum_{i=1}^{N}\left[D(i)-M_{D}\right]^{3}}{\left\{\frac{1}{N-1} \sum_{i=1}^{N}\left[D(i)-M_{D}\right]^{2}\right\}^{3 / 2}}$

It can have either negative values, which means it leans towards the right side of the Gaussian distribution, or positive values, meaning that it leans towards the left side of the Gaussian distribution - see Figure SA2.

Kurtosis is a measure of the amplitude with respect to the normal probability distribution and it can be calculated as:

$\gamma_{2}=\frac{\frac{1}{N} \sum_{i=1}^{N}\left[D(i)-M_{D}\right]^{4}}{\left\{\frac{1}{N-1} \sum_{i=1}^{N}\left[D(i)-M_{D}\right]^{2}\right\}^{2}}$

The kurtosis value of a normal distribution is 3. Distributions for which kurtosis is higher than 3 are called leptokurtic and have nearer extreme values, the graphic being "taller" than in the case of a normal Gaussian distribution. When kurtosis has values lower than 3, the distributions are called platykurtic and have the property that they are asymptotically closing to zero much harder than a normal distribution, so that their extremes are farther away, and the graphic is "shorter" - see Figure SA3.

The histogram is a graphic method used for synthetizing continuously or discrete recorded data, measured on a certain time interval. It pictures the major characteristics of signal's data distribution. A histogram divides the possible signal's values domain in classes or groups. In Figure SA4 we 


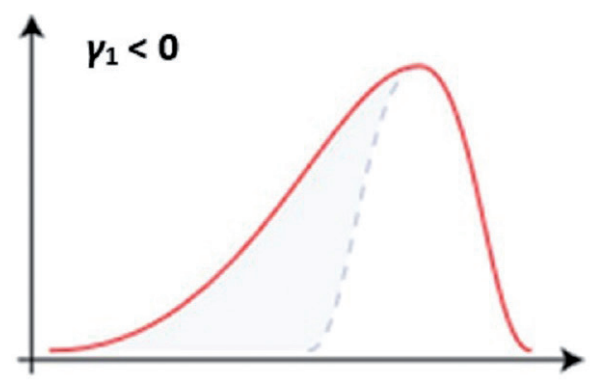

Figure SA2.

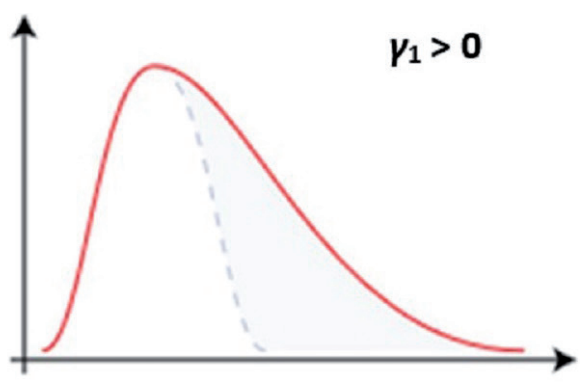

give some examples of histograms corresponding to typical distributions (Arce 2004):

i. normal distribution (Fig. SA3a) - it is described by a bellshaped curve (Gauss curve); its maximum frequency is in the center;

ii. bimodal distribution (Fig. SA3b) - it shows two maximums and indicates that the data comes from multiple simultaneous processes;

iii. truncated distribution (Fig. SA3c) - can be characterized by a steep decrease at one end, indicating a possible prior sorting;

iv. ragged plateau distribution (Fig. SA3d) - it has the aspect of alternating ridges, indicating a possible measurement error;

v. skewed distribution (Fig. SA4e) - it looks like an asymmetric curve, with values tending to concentrate at one end.

The histograph is a graphic formed by connecting segments from the upper parts of histogram's rectangles (Figure SA5). A histograph smoothens abrupt changes that can appear in a histogram.

\section{Signal analysis using nonlinear dynamics methods}

Nonlinear dynamics proposes a vast array of methods for signal analysis. Selecting one is done taking into account the signal specifics and the information one wants to obtain. We will mention only the methods employed in this work.

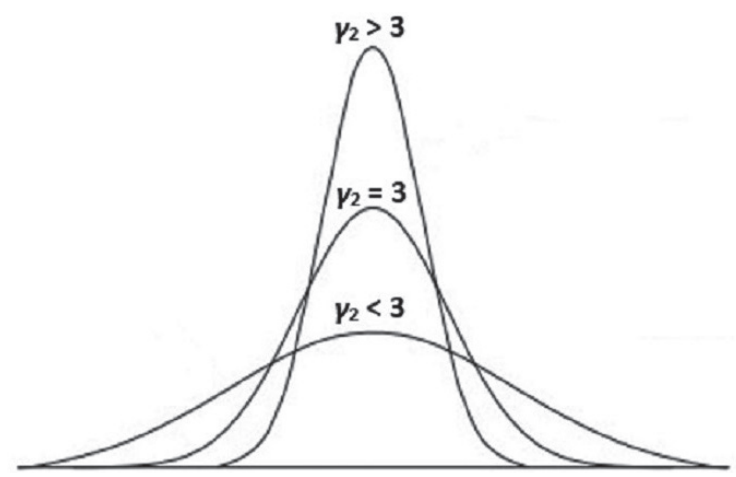

Figure SA3.

The Lyapunov exponents associated to a phase space trajectory of a dynamic system are a measure of the average expansion or contraction rate of trajectories around it, i.e. trajectories that arise from very close initial conditions (Arce 2004). These exponents are asymptotic quantities, locally defined as follows. Let us consider $x(t)$ the trajectory in the phase space of a dynamic system, which starts from an initial condition very close to $x_{0}: x^{\prime}(t=0)=x_{0}$. We define the distance between the two trajectories as $y(t)=\left|x(t)-x^{\prime}(t)\right|$. Considering that this distance varies exponentially with time (this hypothesis must be verified for each case in particular):

$$
y(t)=y(0) e^{\lambda t}
$$

The exponent, which appears in this relation, $\lambda$ is called the Lyapunov exponent. If the phase space is $n$-dimensional (there are $n$ independent quantities describing the state of the system),
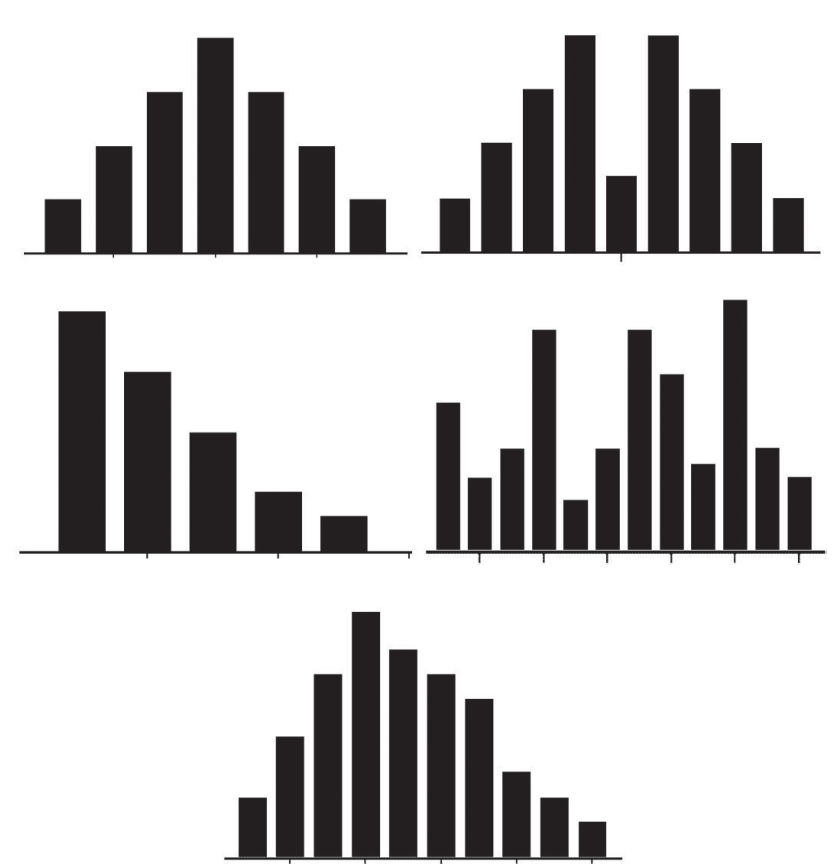

Figure SA4. 


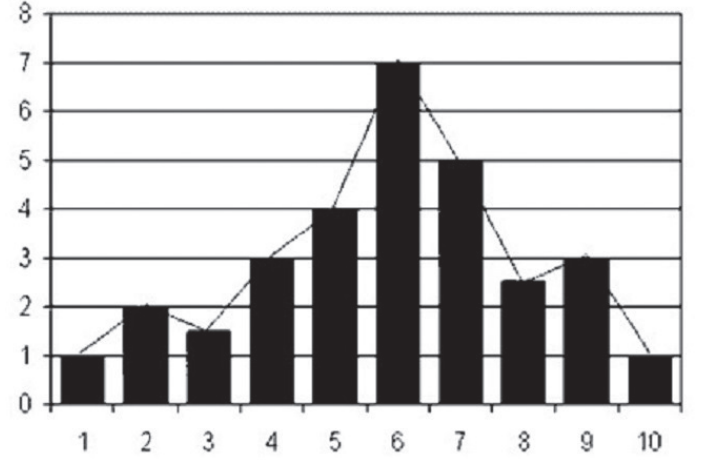

Figure SA5.

then we can define a Lyapunov exponent for each dimension, therefore $n$ Lyapunov coefficients exist, which, together, form the Lyapunov spectrum (Nayfeh and Balachandran 2004). If the Lyapunov exponent is positive, it means that there is a high divergence of initial trajectories from neighboring initial conditions, this being characteristic to chaotic dynamic systems. We usually don't need to calculate all the Lyapunov exponents, because there is a fast algorithm for calculating the highest Lyapunov exponent. If this is positive, the system is chaotic; if it is negative, it means that all the other Lyapunov exponents are negative; therefore the system is not chaotic.

The phase space for a system dynamic is usually higher than 3, so that its graphical representation is impossible. This type of spaces can only be visualized by using projection on subspaces of 3 dimension. Eckman, Kamphorst, and Ruelle developed a method for visualizing the recurrence of state $x_{i}$, in a phase space (Eckmann et al. 1987). Their method allows investigating the system's trajectory in a $m$-dimensional space by representing its two-dimensional recurrences. The recurrence of a state corresponding to the moment $t_{t}$ at a different time $t_{j}$ can be graphically presented by a two-dimensional square matrix, in which the dark colored points represent the recurrence. This is called a recurrence graphic, and it can be defined by the following relation:

$$
\begin{aligned}
& R(i, j)=\Theta(\varepsilon-\|\vec{r}(i)-\vec{r}(j)\|), \quad \vec{r}(i) \in R^{m}, \\
& i, j=1, \ldots, N
\end{aligned}
$$

where $N$ is the number of $x_{i}$ states considered, $\varepsilon$ is a threshold distance, and $\Theta$ is the Heaviside step function (Arce 2004). The recurrence graphic shows both large scale (topology) and reduced scale (texture) characteristics. Topology offers a global impression, which can be characterized as homogenous, period, drift, or disrupted. Textures form the base of the recurrence graphic qualitative analysis. Aflori and Dimitriu (2006) devised a summative table for a qualitative interpretation of recurrence graphics - see Table SA1.

In Figure SA6, recurrence maps are drawn, corresponding to "white" noise (random signal), harmonic oscillations (perfect sinusoidal), Brownian motion, and to a healthy patient's ECG (Perc 2005).

For the quantitative estimation of the structural degree of the recurrence map, the spatial-temporal entropy is calculated, measuring both the spatial and temporal structural degrees. It compares the global distribution of colors along the whole recurrence map with the distribution of colors along each diagonal of the map (Aflori and Dimitriu 2006). The result is normed and presented in the form of a maximum value percentage, corresponding to a random signal. A 100\% spatial-temporal entropy indicates the absence of any structure (uniform color distribution), while a value of $0 \%$ for the same entropy means a perfect structuralizing (distinct model in the map, total predictability) (Aflori and Dimitriu 2006).

In most experimental situations it is very hard to establish the independent coordinates that describe a systems' dynamics, necessary for building the phase space. For this reason, several alternative methods for constructing such a space have been developed, the most well-known one being the delay time method.

Table SA1. Qualitative interpretation of recurrence graphics

\begin{tabular}{ll}
\hline Observations & Interpretation \\
\hline Homogeneity & The process is stationary \\
Upper left and lower right decoloring & The process is not stationary, it contains a trend or a drift \\
$\begin{array}{l}\text { Disruption (white bands) } \\
\text { Periodic patterns }\end{array}$ & The process is not stationary; some states are rare or far from normal; transitions occurred \\
Isolated singular points & Cyclicalities in the process; the temporal distance between period patterns corresponds to the period \\
$\begin{array}{l}\text { Diagonal lines (parallel with the } \\
\text { main diagonal) }\end{array}$ & $\begin{array}{l}\text { States evolutions is similar at different times; the process can be a deterministic one; if the diagonal } \\
\text { lines appear between isolated singular points, the process could be chaotic; if, in addition, the }\end{array}$ \\
$\begin{array}{ll}\text { Diagonal lines (perpendicular on the } \\
\text { main diagonal) }\end{array}$ & $\begin{array}{l}\text { States evolution is similar at different time intervals but with inverse time; sometimes this indicates } \\
\text { Vertical or horizontal lines/clusters }\end{array}$ \\
\hline
\end{tabular}



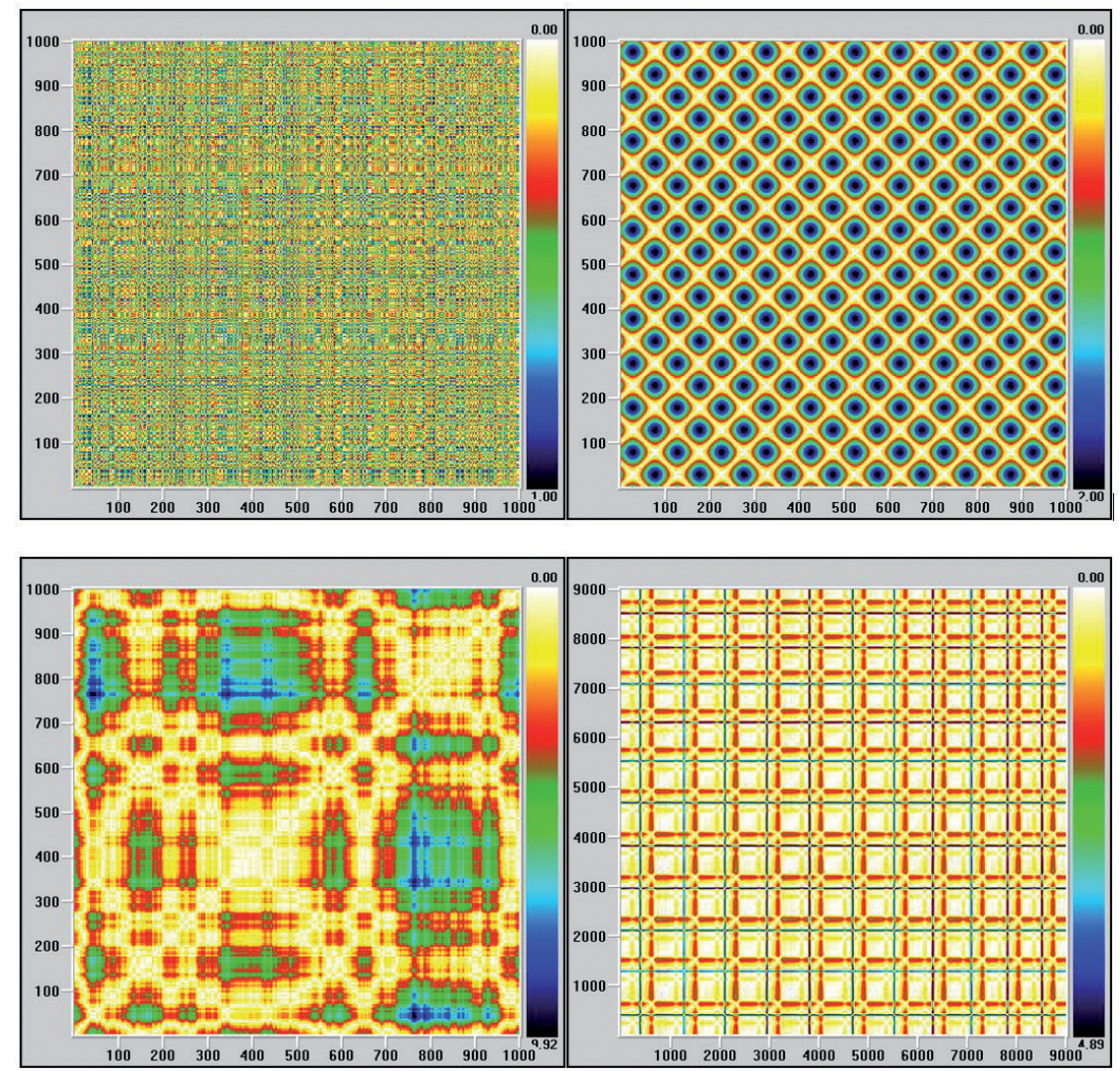

Figure SA6.

Experimentally, most often a single system variable is measured:

$s_{n}=s(t)=s\left(t_{0}+n \tau_{s}\right)$

where $\tau_{s}$ is the sampling time of the instrument used for measuring the $s$ variable. A series of paper written at the end of the $20^{\text {th }}$ century suggested that the measurement of a single signal could be sufficient to "reconstruct" a phase space in which the system's dynamics is equivalent with the one from the original phase space. Packard et al. (1980) demonstrated this equivalency through numerical simulations, while Takens (1981) demonstrated it with mathematical rigor. As a consequence of this equivalence, the attractor from the reconstructed phase space has the same invariants (like the Lyapunov exponents) as the original one.

In order to reconstruct the independent coordinates of the phase space, Packard and co. proposed using the signal's derivatives (Packard et al. 1980). In this way, the derivates can be approximated with the finite differences:

$\frac{d s}{d t}\left(t_{0}+n \tau_{s}\right) \cong \frac{s\left(t_{0}+n \tau_{s}\right)-s\left[t_{0}+(n-1) \tau_{s}\right]}{\tau_{s}}$

$$
\begin{aligned}
& \frac{d^{2} s}{d t^{2}}\left(t_{0}+n \tau_{s}\right) \cong \frac{s\left[t_{0}+(n+1) \tau_{s}\right]-2 s\left(t_{0}+n \tau_{s}\right)}{\tau_{s}^{2}}+ \\
& +\frac{s\left[t_{0}+(n-1) \tau_{s}\right]}{\tau_{s}^{2}}
\end{aligned}
$$

From the above-written formulas it can be observed that, at each new differentiation, new information already contained in the measured signal is added, but at time moments delayed with a multiple of the delay time. This observation lead Packard et al. (1980), Takens (1981), and Ruelle (1989) to the conclusion that there is no need for derivatives in order to compose a coordinates system that describes the structure of orbits from the phase space. Instead, one can directly use the $s(t+n \tau)$ variables, where $n=1,2, \ldots, d$, and $\tau=k \tau_{s}$ is an appropriately chosen delay time. We can define, thus, the so-called delayed coordinates vectors as:

$y_{n}=\left\{s\left(t_{0}+n \tau_{s}\right), s\left(t_{0}+n \tau_{s}+k \tau_{s}\right), \ldots\right.$,

$\left.s\left[t_{0}+n \tau_{s}+k(d-1) \tau_{s}\right]\right\}^{T}$ 
$y_{n}=\left\{s_{n}, s_{n+k}, s_{n+2 k}, \ldots, s_{n+(d-1) k}\right\}^{T}$

The space constructed with the $y_{n}$ vectors is called the reconstructed phase space. According to Takens (1981) and Mané (1981), the geometrical structure for the dynamics of the systems for which the variable $s$ has been measured can be observed in the reconstructed $d$-dimensional Euclidian space, if $d \geq 2 d_{a}+1$, where $d_{a}$ is the attractor's dimension. In 1991, Sauer et al. (1991) had shown that it is sufficient for $d \geq 2 d_{a}$. The parameter $\tau$ is called the delay time, and the integer $d$ is called the immersion dimension.

The immersion dimension must be large enough so that no intersection of orbits reconstructed with themselves (false intersections) should appear. There are several ways for determining this dimension; the most often used one being the systems' invariants saturations and the false nearest neighbors method (Nayfeh and Balachandran 2004).

In theory, the delay time can be arbitrary chosen if we have infinity of data, not altered by noise. As this is improbable in practice, the value of the delay time must be chosen with care. If this value is too low, the trajectories in the reconstructed phase space will clutter towards the main diagonal, due to the fact that the delayed coordinates are excessively correlated. On the other hand, if the chosen value is too high, an artificial decorrelation can appear, and the delayed coordinates become uncorrelated. The most often used methods for determining the delay time are the autocorrelation function, the average mutual information, and the generalized correlations integral methods (Nayfeh and Balachandran 2004).

\section{References}

Aflori M, Dimitriu DG (2006): Signal analysis using nonlinear dynamics methods (in Romanian). In: Physics complements for PhD candidates (in Romanian), vol. I (Eds. Dumitrascu L, Dumitrascu I, Dorohoi DO, Dimitriu DG, Apreotesei G, Aflori M), Tehnopress, Iasi

Arce GR (2004): Nonlinear Signal Processing: A Statistical Approach. Wiley, Hoboken https://doi.org/10.1002/0471691852

Eckmann JP, Kamphorst SO, Ruelle D (1987): Recurrence plots of dynamical systems. Europhysics Letters 4, 973-977 https://doi.org/10.1209/0295-5075/4/9/004

Mané R (1981): On the dimension of compact invariant sets of certain nonlinear maps. Lecture Notes in Mathematics 898, 230-242 https://doi.org/10.1007/BFb0091916

Nayfeh AH, Balachandran B (2004): Applied Nonlinear Dynamics. Analytical, Computational, and Experimental Methods. Wiley-VCH, Weinheim

Packard NH, Crutchfield JP, Farmer JD, Shaw RS (1980): Geometry from a time series. Physical Review Letters 45, 712-716 https://doi.org/10.1103/PhysRevLett.45.712
Perc M (2005): Nonlinear time series analysis of the human electrocardiogram. European Journal of Physics 26, 757-768 https://doi.org/10.1088/0143-0807/26/5/008

Ruelle D (1989): Chaotic Evolution and Strange Attractors. Cambridge University Press, Cambridge https://doi.org/10.1017/CBO9780511608773

Sauer T, Yorke JA, Casdagli M (1991): Embedology. Journal of Statistical Physics 65, 579-616 https://doi.org/10.1007/BF01053745

Takens F (1981): Detecting strange attractors in turbulence. Lecture Notes in Mathematics 898, 366-381 https://doi.org/10.1007/BFb0091924

\section{Section B}

In this supplementary material we have selected another 4 ECG signals from the PhysioBank database and calculated the same statistical parameters as in the case presented in the main body of this paper.

For statistically correlating the results we have used Google Colab ${ }^{\circledR}$.

Colaboratory, or "Colab" for short, allows you to write and execute Python in the browser, with zero configuration required, free access to GPUs, and also easy sharing.

In the following we want to present the program input and output for all 5 analyzed ECG signals.

i) Output data for the 5 analyzed signals - pre-crisis.

import numpy as np

import pandas as pd

import matplotlib.pyplot as plt

import seaborn as sns

$\mathrm{df}=$ pd.read_csv('pre-crisis.csv')

df.info()

\section{OUT}

<class 'pandas.core.frame.DataFrame'>

RangeIndex: 5 entries, 0 to 4

Data columns (total 6 columns):

\# Column Non-Null Count Dtype

0 R-R interval median 5 non-null float64

1 Variance 5 non-null float64

2 Geometric standard deviation 5 non-null float64

3 Skewness 5 non-null float64

4 Kurtosis 5 non-null float64

5 Largest Lyapunov exponent 5 non-null float64 dtypes: float64(6) memory usage: 368.0 bytes

df.describe() 


\section{OUT}

\begin{tabular}{lcccccc}
\hline & $\begin{array}{c}\text { 1/R-R interval } \\
\text { median }\end{array}$ & Variance & $\begin{array}{c}\text { Geometric } \\
\text { standard deviation }\end{array}$ & Skewness & Kurtosis & $\begin{array}{c}\text { Largest Lyapunov } \\
\text { exponent }\end{array}$ \\
\hline count & 5.000000 & 5.00000 & 5.000000 & 5.00000 & 5.000000 & 5.000000 \\
mean & 58.986060 & 23.21976 & 1.089440 & 8.45328 & 81.316440 & 0.013928 \\
std & 2.679212 & 0.49851 & 0.033421 & 0.16355 & 1.684059 & 0.000815 \\
min & 55.936400 & 22.73340 & 1.054500 & 8.25090 & 78.946800 & 0.012832 \\
$\mathbf{2 5 \%}$ & 56.603700 & 22.87100 & 1.068400 & 8.34160 & 80.459500 & 0.013367 \\
$\mathbf{5 0 \%}$ & 59.824400 & 23.00600 & 1.076600 & 8.46670 & 81.385500 & 0.014120 \\
$\mathbf{7 5 \%}$ & 60.168700 & 23.60260 & 1.112200 & 8.53960 & 82.845500 & 0.014529 \\
$\mathbf{m a x}$ & 62.397100 & 23.88580 & 1.135500 & 8.66760 & 82.944900 & 0.014791 \\
\hline
\end{tabular}

corr_matrix $=$ df.corr ()

plt.figure $($ figsize $=(20,15))$

sns.heatmap(corr_matrix, annot=True)

plt.show()

\section{OUT}

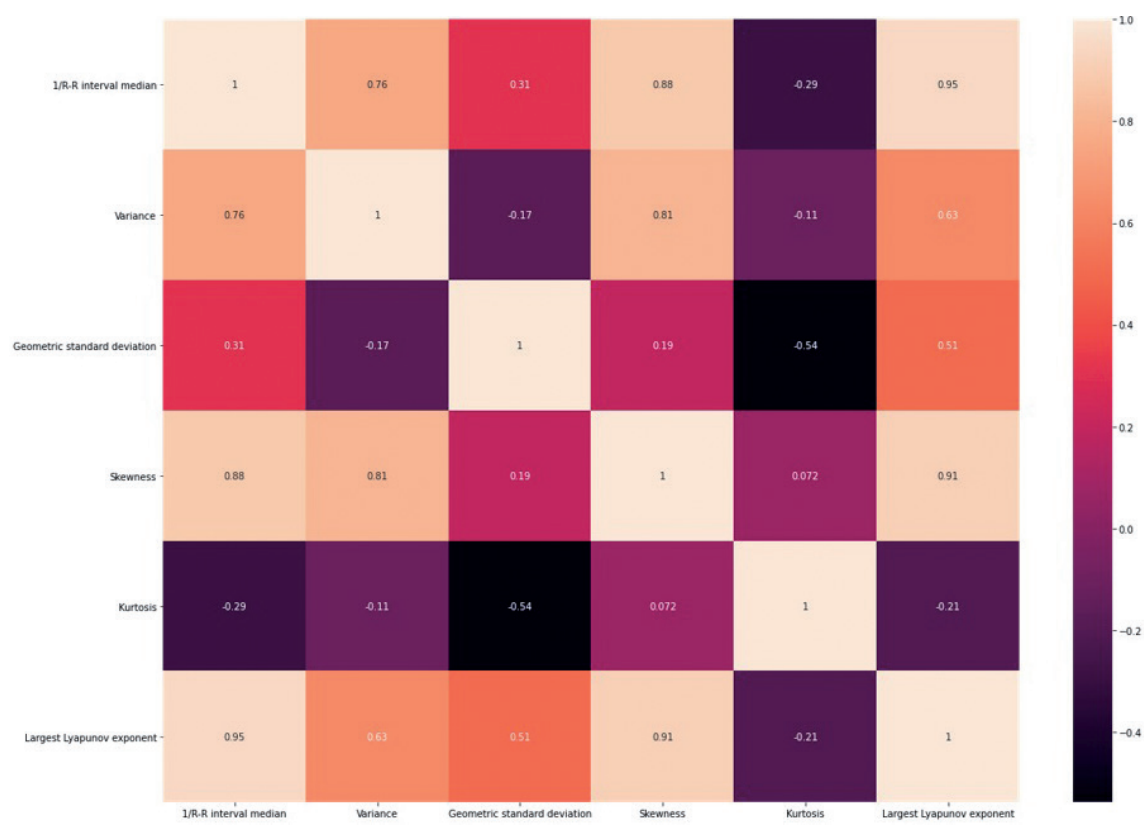

ii) Output data for the 5 analyzed signals - AFIB 1. import numpy as $n p$ import pandas as pd import matplotlib.pyplot as plt import seaborn as sns $\mathrm{df}=$ pd.read_csv('afib1.csv')

df.info()

\section{OUT}

<class 'pandas.core.frame.DataFrame'> RangeIndex: 5 entries, 0 to 4
Data columns (total 6 columns): \# Column Non-Null Count Dtype

0 R-R interval median 5 non-null float64

1 Variance 5 non-null float64

2 Geometric standard deviation 5 non-null float64

3 Skewness 5 non-null float 64

4 Kurtosis 5 non-null float64

5 Largest Lyapunov exponent 5 non-null float64 dtypes: float64(6)

memory usage: 368.0 bytes

df.describe() 


\section{OUT}

\begin{tabular}{|c|c|c|c|c|c|c|}
\hline & $\begin{array}{l}\text { 1/R-R interval } \\
\text { median }\end{array}$ & Variance & $\begin{array}{c}\text { Geometric } \\
\text { standard deviation }\end{array}$ & Skewness & Kurtosis & $\begin{array}{c}\text { Largest Lyapunov } \\
\text { exponent }\end{array}$ \\
\hline count & 5.000000 & 5.000000 & 5.000000 & 5.000000 & 5.000000 & 5.000000 \\
\hline mean & 60.602700 & 712.231360 & 1.471800 & 0.778020 & -1.107200 & 0.212341 \\
\hline std & 9.246203 & 5.563042 & 0.041145 & 0.007043 & 0.021615 & 0.007595 \\
\hline $\min$ & 52.785200 & 705.713800 & 1.430900 & 0.766300 & -1.142000 & 0.199779 \\
\hline $25 \%$ & 53.380700 & 707.124900 & 1.439400 & 0.776600 & -1.114000 & 0.211145 \\
\hline $50 \%$ & 58.964100 & 714.337600 & 1.462400 & 0.781400 & -1.098000 & 0.215325 \\
\hline $75 \%$ & 62.348800 & 715.331200 & 1.496700 & 0.782900 & -1.092000 & 0.216212 \\
\hline $\max$ & 75.534700 & 718.649300 & 1.529600 & 0.782900 & -1.090000 & 0.219243 \\
\hline
\end{tabular}

corr_matrix $=$ df.corr ()

plt.figure $($ figsize $=(20,15))$

sns.heatmap(corr_matrix, annot=True)

plt.show()

\section{OUT}

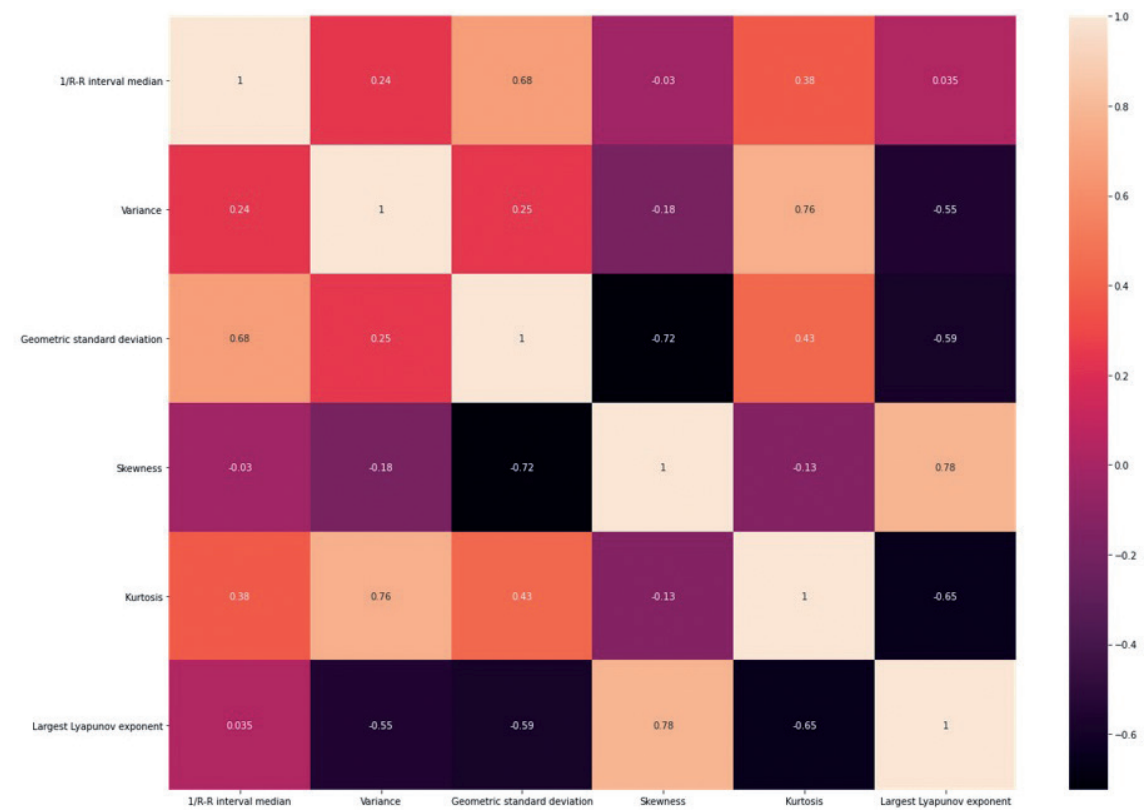

iii) Output data for the 5 analyzed signals - AFL. import numpy as np import pandas as pd import matplotlib.pyplot as plt import seaborn as sns df $=$ pd.read_csv('afl.csv')

df.info()

\section{OUT}

<class 'pandas.core.frame.DataFrame'> RangeIndex: 5 entries, 0 to 4
Data columns (total 6 columns): \# Column Non-Null Count Dtype

0 R-R interval median 5 non-null float64

1 Variance 5 non-null float64

2 Geometric standard deviation 5 non-null float64

3 Skewness 5 non-null float 64

4 Kurtosis 5 non-null float64

5 Largest Lyapunov exponent 5 non-null float64 dtypes: float64(6)

memory usage: 368.0 bytes

df.describe() 


\section{OUT}

\begin{tabular}{lcccccc}
\hline & $\begin{array}{c}\text { 1/R-R interval } \\
\text { median }\end{array}$ & Variance & $\begin{array}{c}\text { Geometric } \\
\text { standard deviation }\end{array}$ & Skewness & Kurtosis & $\begin{array}{c}\text { Largest Lyapunov } \\
\text { exponent }\end{array}$ \\
\hline count & 5.00000 & 5.000000 & 5.000000 & 5.000000 & 5.000000 & 5.000000 \\
mean & 123.60302 & 18.232860 & 1.230100 & -0.036260 & 0.462680 & 0.081734 \\
std & 6.91226 & 0.389249 & 0.013911 & 0.000541 & 0.005615 & 0.003371 \\
min & 115.38460 & 17.865100 & 1.210500 & -0.037100 & 0.457800 & 0.076186 \\
$\mathbf{2 5 \%}$ & 118.92740 & 17.991100 & 1.222600 & -0.036400 & 0.458600 & 0.081154 \\
$\mathbf{5 0 \%}$ & 122.45710 & 18.012600 & 1.234700 & -0.036200 & 0.462000 & 0.082811 \\
$\mathbf{7 5 \%}$ & 129.66380 & 18.548800 & 1.235900 & -0.035900 & 0.463100 & 0.083804 \\
$\mathbf{m a x}$ & 131.58220 & 18.746700 & 1.246800 & -0.035700 & 0.471900 & 0.084715 \\
\hline
\end{tabular}

corr_matrix $=$ df.corr ()

plt.figure $($ figsize $=(20,15))$

sns.heatmap(corr_matrix, annot=True)

plt.show()

\section{OUT}

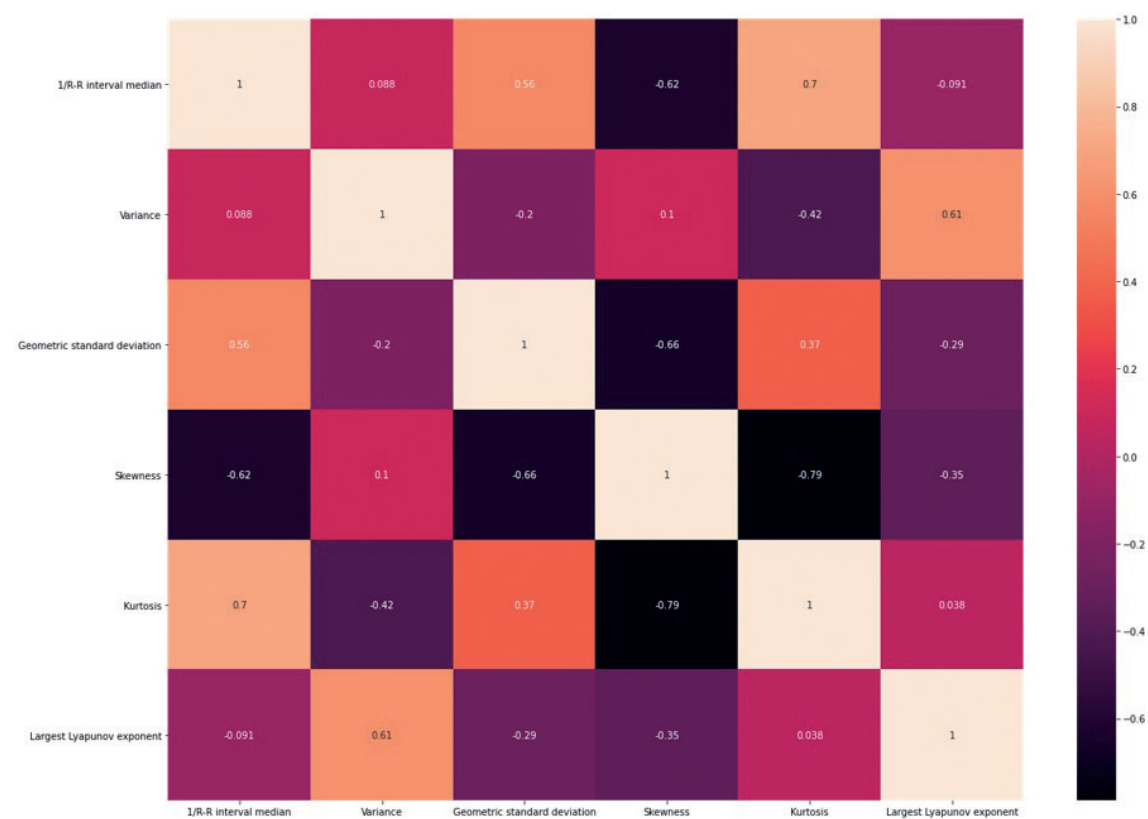

iv) Output data for the 5 analyzed signals - AFIB 2. import numpy as np import pandas as pd import matplotlib.pyplot as plt import seaborn as sns $\mathrm{df}=$ pd.read_csv('afib2.csv')

df.info()

\section{OUT}

<class 'pandas.core.frame.DataFrame'> RangeIndex: 5 entries, 0 to 4
Data columns (total 6 columns): \# Column Non-Null Count Dtype

0 R-R interval median 5 non-null float64

1 Variance 5 non-null float64

2 Geometric standard deviation 5 non-null float 64

3 Skewness 5 non-null float 64

4 Kurtosis 5 non-null float64

5 Largest Lyapunov exponent 5 non-null float64 dtypes: float64(6)

memory usage: 368.0 bytes

df.describe() 


\section{OUT}

\begin{tabular}{lrrcccc}
\hline & $\begin{array}{c}\text { R-R interval } \\
\text { median }\end{array}$ & Variance & $\begin{array}{c}\text { Geometric } \\
\text { standard deviation }\end{array}$ & Skewness & Kurtosis & $\begin{array}{c}\text { Largest Lyapunov } \\
\text { exponent }\end{array}$ \\
\hline count & 5.00000 & 5.000000 & 5.000000 & 5.000000 & 5.000000 & 5.000000 \\
mean & 81.70676 & 399.133560 & 1.275680 & 0.717940 & 0.046140 & 0.127618 \\
std & 5.94071 & 9.456367 & 0.021265 & 0.009775 & 0.002712 & 0.010979 \\
min & 75.82670 & 388.067400 & 1.247200 & 0.704700 & 0.042100 & 0.115384 \\
$\mathbf{2 5 \%}$ & 76.92310 & 391.197000 & 1.266200 & 0.713100 & 0.045600 & 0.117860 \\
$\mathbf{5 0 \%}$ & 81.28340 & 400.976900 & 1.275600 & 0.719400 & 0.045700 & 0.127831 \\
$\mathbf{7 5 \%}$ & 83.92850 & 404.425200 & 1.285100 & 0.721600 & 0.048600 & 0.138368 \\
$\mathbf{m a x}$ & 90.57210 & 411.001300 & 1.304300 & 0.730900 & 0.048700 & 0.138646 \\
\hline
\end{tabular}

corr_matrix $=$ df.corr ()

plt.figure $($ figsize $=(20,15))$

sns.heatmap(corr_matrix, annot=True)

plt.show()

\section{OUT}

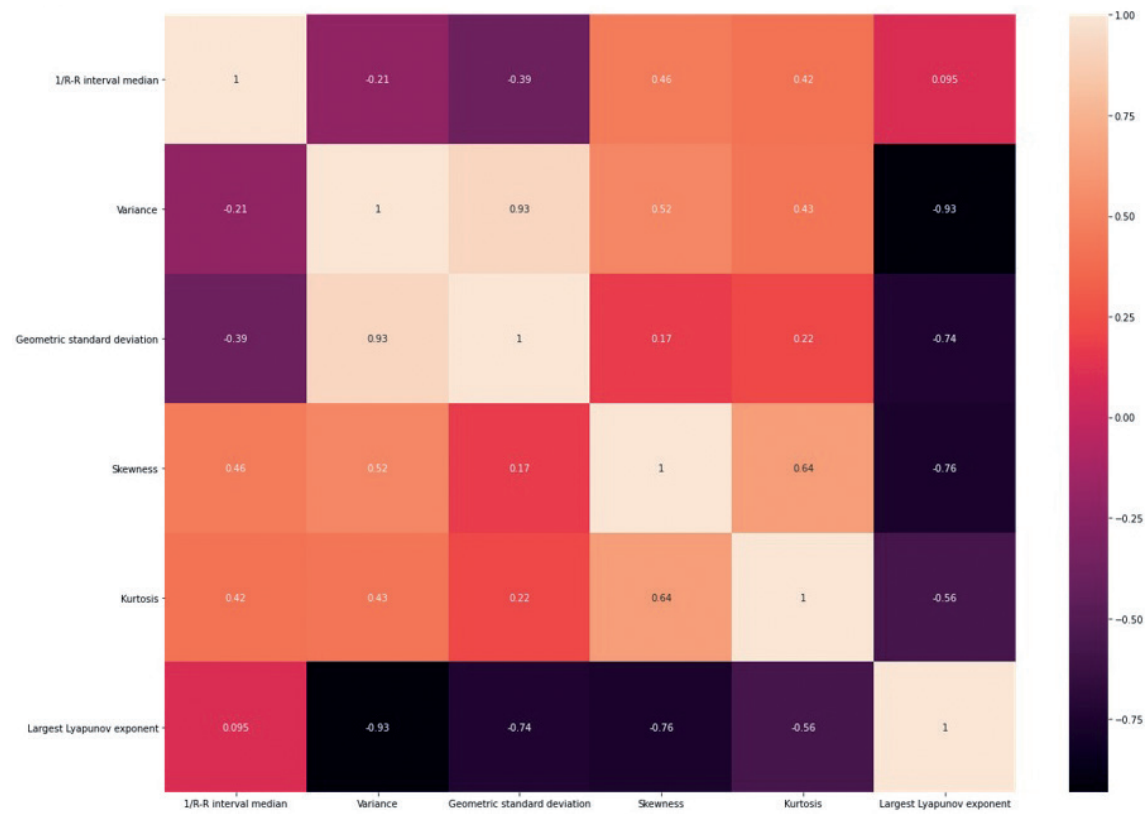

v) Output data for the 5 analyzed signals - post-crisis. import numpy as $\mathrm{np}$ import pandas as pd import matplotlib.pyplot as plt import seaborn as sns $\mathrm{df}=$ pd.read_csv('post-crisis.csv')

df.info()

\section{OUT}

<class 'pandas.core.frame.DataFrame'> RangeIndex: 5 entries, 0 to 4
Data columns (total 6 columns): \# Column Non-Null Count Dtype

0 R-R interval median 5 non-null float64

1 Variance 5 non-null float64

2 Geometric standard deviation 5 non-null float64

3 Skewness 5 non-null float 64

4 Kurtosis 5 non-null float64

5 Largest Lyapunov exponent 5 non-null float64 dtypes: float64(6)

memory usage: 368.0 bytes

df.describe() 


\section{OUT}

\begin{tabular}{|c|c|c|c|c|c|c|}
\hline & $\begin{array}{l}\text { R-R interval } \\
\text { median }\end{array}$ & Variance & $\begin{array}{c}\text { Geometric } \\
\text { standard deviation }\end{array}$ & Skewness & Kurtosis & $\begin{array}{c}\text { Largest Lyapunov } \\
\text { exponent }\end{array}$ \\
\hline count & 5.000000 & 5.00000 & 5.000000 & 5.00000 & 5.000000 & 5.000000 \\
\hline mean & 58.986060 & 23.21976 & 1.089440 & 8.45328 & 81.316440 & 0.013928 \\
\hline std & 2.679212 & 0.49851 & 0.033421 & 0.16355 & 1.684059 & 0.000815 \\
\hline $\min$ & 55.936400 & 22.73340 & 1.054500 & 8.25090 & 78.946800 & 0.012832 \\
\hline $25 \%$ & 56.603700 & 22.87100 & 1.068400 & 8.34160 & 80.459500 & 0.013367 \\
\hline $50 \%$ & 59.824400 & 23.00600 & 1.076600 & 8.46670 & 81.385500 & 0.014120 \\
\hline $75 \%$ & 60.168700 & 23.60260 & 1.112200 & 8.53960 & 82.845500 & 0.014529 \\
\hline $\max$ & 62.397100 & 23.88580 & 1.135500 & 8.66760 & 82.944900 & 0.014791 \\
\hline
\end{tabular}

corr_matrix $=$ df.corr ()

plt.figure $($ figsize $=(20,15))$

sns.heatmap(corr_matrix, annot=True)

plt.show()

\section{OUT}

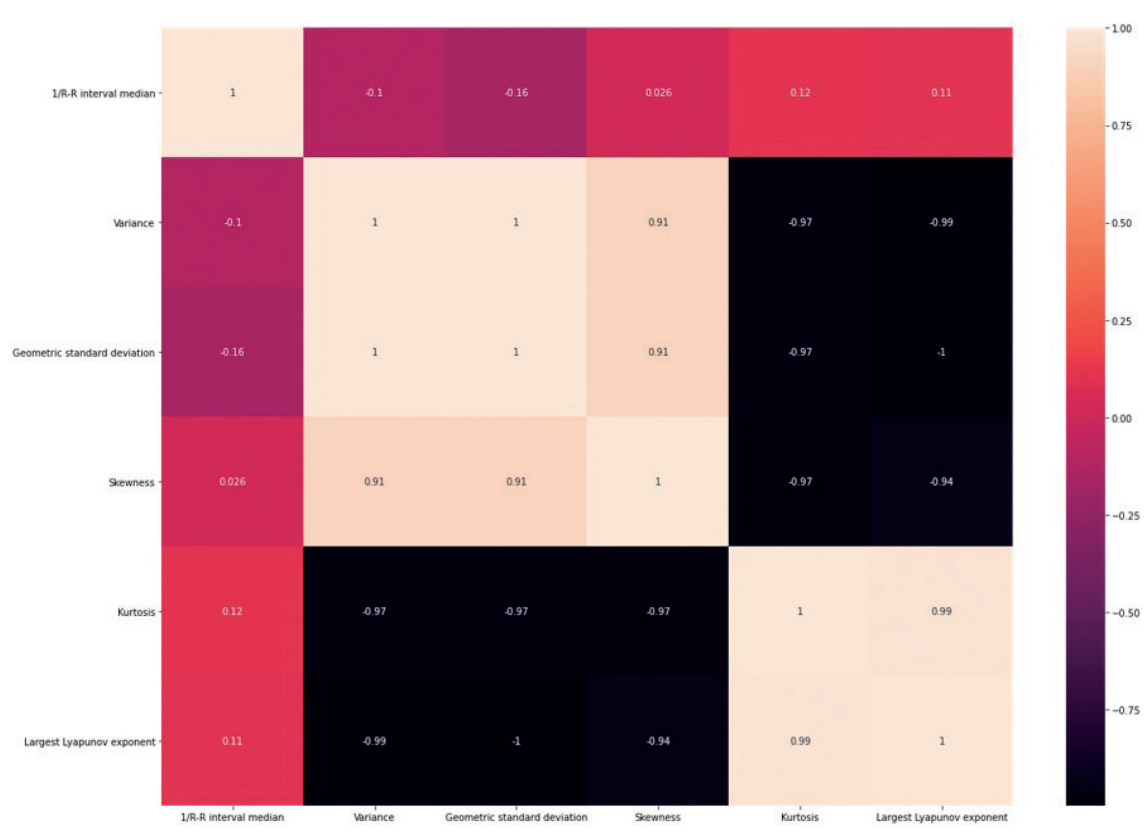

As it can be seen from the above-presented data, strong $(>0.75)$ or very strong $(>0.9)$ correlations have been found for:

i) Pre-crisis:

Largest Lyapunov exponent - 1/R-R interval median correlation: 0.95

Largest Lyapunov exponent - Skewness correlation: 0.91

Skewness - 1/R-R interval median correlation: 0.88

Skewness - Variance correlation: 0.81

Variance - 1/R-R interval median correlation: 0.76

ii) AFIB1:

Largest Lyapunov exponent - Skewness correlation: 0.78

Kurtosis - Variance correlation correlation: 0.76
Skewness - Geometric standard deviation correlation: -0.72 iii) AFL:

Kurtosis - Skewness: -0.79

iv) AFIB2:

Largest Lyapunov exponent - Variance correlation: -0.93 Largest Lyapunov exponent - Geometric standard deviation correlation: -0.74

Largest Lyapunov exponent - Skewness correlation: -0.76 Geometric standard deviation - Variance correlation: 0.93

v) Post-crisis:

Largest Lyapunov exponent - Variance correlation: -0.99 Largest Lyapunov exponent - Geometric standard deviation correlation: -1 
Largest Lyapunov exponent - Skewness correlation: -0.94

Largest Lyapunov exponent - Kurtosis correlation: 0.99

Kurtosis - Variance correlation: -0.97

Kurtosis - Geometric standard deviation correlation: $-0.97$
Kurtosis - Skewness correlation: -0.97

Skewness - Variance correlation: 0.91

Skewness - Geometric standard deviation correlation: 0.91

Geometric standard deviation correlation - Variance: 1 